Atmos. Chem. Phys., 13, 2115-2132, 2013

www.atmos-chem-phys.net/13/2115/2013/

doi:10.5194/acp-13-2115-2013

(C) Author(s) 2013. CC Attribution 3.0 License.

\title{
The importance of low-deformation vorticity in tropical cyclone formation
}

\author{
K. J. Tory, R. A. Dare, N. E. Davidson, J. L. McBride, and S. S. Chand \\ Centre for Australian Weather and Climate Research, Melbourne, Australia \\ Correspondence to: K. J. Tory (k.tory@bom.gov.au) \\ Received: 12 April 2012 - Published in Atmos. Chem. Phys. Discuss.: 18 July 2012 \\ Revised: 16 January 2013 - Accepted: 20 January 2013 - Published: 22 February 2013
}

\begin{abstract}
Studies of tropical cyclone (TC) formation from tropical waves have shown that $\mathrm{TC}$ formation requires a wave-relative quasi-closed circulation: the "marsupial pouch" concept. This results in a layerwise nearly contained region of atmosphere in which the modification of moisture, temperature and vorticity profiles by convective and boundary layer processes occurs undisturbed. The pouch concept is further developed in this paper. TCs develop near the centre of the pouch where the flow is in near solid body rotation. A reference-frame independent parameter is introduced that effectively measures the level of solid-body rotation in the lower troposphere. The parameter is the product of a normalized Okubo-Weiss parameter and absolute vorticity (OWZ).

Using $20 \mathrm{yr}$ of ERA-interim reanalysis data and the IBTrACS global TC database, it is shown $95 \%$ of TCs including, but not limited to, those forming in tropical waves are associated with enhanced levels of OWZ on both the 850 and $500 \mathrm{hPa}$ pressure levels at the time of TC declaration, while $90 \%$ show enhanced OWZ for at least $24 \mathrm{~h}$ prior to declaration. This result prompts the question of whether the pouch concept extends beyond wave-type formation to all TC formations world-wide.

Combining the OWZ with a low vertical shear requirement and lower troposphere relative humidity thresholds, an imminent genesis parameter is defined. The parameter includes only relatively large-scale fluid properties that are resolved by coarse grid model data $(>150 \mathrm{~km})$, which means it can be used as a TC detector for climate model applications. It is also useful as a cyclogenesis diagnostic in higher resolution models such as real-time global forecast models.
\end{abstract}

\section{Introduction}

Tropical cyclones (TCs) form in oceanic regions of enhanced cyclonic vorticity and humidity. Tropical waves, monsoon troughs, extratropical disturbances, and topographical flows provide environments of enhanced lower-tropospheric cyclonic vorticity, where convection is favoured, within which TCs may form. Such environments are necessary but not sufficient for TC formation.

The fundamental system-scale spin-up mechanism during TC formation is the vorticity concentration (enhancement) associated with mass convergence in the low to middle troposphere, in response to a sustained convectively driven upward mass flux. In typical tropical convection the drivers of vertical mass flux, namely condensational heating (producing positive buoyancy and ascent) and evaporative cooling (producing negative buoyancy and reduced ascent, or descent) are in competition when averaged over storm systems such as convective clusters and mesoscale convective systems. While the upward mass flux typically dominates over the storm system life-time (e.g., Mapes and Houze, 1992, 1993, 1995), the net convergence is relatively weak. Modelling studies suggest the convection in developing TCs, on the other hand, is dominated by intense updrafts and comparatively weak downdrafts (e.g., Nolan, 2007).

The typical tropical oceanic thermodynamic environment has a minimum in equivalent potential temperature $\left(\theta_{\mathrm{e}}\right)$ or moist static energy in the middle troposphere (e.g., Riehl and Malkus, 1958; Gray, 1973; Yanai et al., 1973; Kingsmill and Houze, 1999). The dry, low $\theta_{\mathrm{e}}$ air in the middle troposphere supports evaporation, which reduces the buoyancy and thus weakens the system upward mass flux, and the lowto middle-troposphere inflow and vorticity convergence in 
convective systems. Evaporation of cloud water or rain reduces updraft intensity and supports convective downdrafts and stratiform subsidence, which weakens or potentially reverses the mean storm-system upward mass flux. A thermodynamic transformation towards a near-saturated environment would appear to be necessary for TC formation, a result supported by recent modelling studies (Nolan, 2007; Wang, 2012 and references therein). Theoretical arguments (Downdraft CAPE, Emanuel, 1994) suggest a vertically well-mixed moist atmosphere may also be necessary to minimise the potential for downdrafts, because even a saturated parcel has downdraft potential in a "moist unstable" environment (Tory and Frank, 2010, p. 67). However, despite the thermodynamic transition towards conditions that reduce evaporation potential and potential for evaporative downdrafts, modeling studies of Nolan (2007) and Wang (2012), have shown that the downdraft mass flux increases as the TC formation process proceeds (although at a much lesser rate than the increasing updraft mass flux). Presumably the increasing availability of moisture to be evaporated, in the intensifying and expanding convection, more than compensates for the less favorable conditions for evaporation and evaporative downdrafts.

One potential mechanism to drive the thermodynamic transformation is the vertical mixing and entrainment and detrainment of the convection itself, which acts to moisten and redistribute the moist entropy more evenly. It might be expected that such a transition could take many hours or days of convective activity (e.g., Zehr, 1992). In that time the convective forcing would need to move with the flow in order to remain focussed on the transforming fluid, and the fluid would need to be protected to avoid contamination from outside dry air throughout the transformation period. This requirement is problematic for wave-type flows (including open waves) in which the convective forcing is attached to the trough, and the fluid on the whole flows through the trough.

A fluid parcel passing through a wave trough experiences convergence that enhances the vorticity as it approaches the trough, and divergence that weakens the vorticity as it departs the trough. The convergence may trigger convection that begins the thermodynamic transformation and further enhances the parcel vorticity, but if the parcel then passes out of the trough the spin-up potential associated with the release of the convective energy (i.e., the increased moistening and rotation) has effectively been lost from the trough. For a successful convectively driven thermodynamic transition the air-mass must be confined to the convective region in a quasi-closed ${ }^{1}$ recirculating flow for many hours or perhaps days. For an initially open wave with convective activity in the trough, the wave must "break" before a quasi-closed circulation and associated recirculating flow develops. More generally, the necessity for wave-relative circular instanta-

\footnotetext{
${ }^{1}$ The circulation is not completely closed. Limited entry and exit of mass is expected.
}

neous flow (if not fully recirculating flow) for TC formation has been recognised for some time. The first sentence of the classic study of Charney and Eliassen (1964) states: "Hurricanes are observed to develop from pre-existing tropical depresssions that somehow have aquired a degree of circular symmetry...”. Additionally, McBride and Zehr (1981) found the high vorticity in TC precursor circulations contained equal contributions, but opposite sign, of zonal and meridional horizontal shear, i.e., highly circular flow.

Dunkerton et al. (2009) identified quasi-closed flow within the breaking wave by constructing streamlines from the wave-relative flow, and established that the flow was recirculating. They identified numerous TCs forming in quasiclosed streamlines, which they likened to the protective pouch of a marsupial. The protective pouch analysis was later used to identify regions of TC formation potential (Wang et al., 2009; Montgomery et al., 2010). The pouch typically contained enhanced vorticity with minimal flow deformation (e.g., flow in near-solid-body rotation) near the pouch centre with increasing flow deformation outwards towards the pouch boundary (Wang et al., 2010a, b; Montgomery et al., 2010). It is within this central pouch region that the thermodynamic transition occurs (Wang, 2012), convection is focussed (Davis and Ahijevych, 2012) and the TC core vortex develops (Dunkerton et al., 2009). The pouch not only protects the developing proto-vortex, but the lowdeformation/enhanced-vorticity flow at the pouch centre is perhaps necessary to support the two processes described in the bottom-up TC formation theory (Hendricks et al., 2004; Montgomery et al., 2006; Tory et al., 2006, 2007), i.e., (i) the aggregation of meso-scale vorticity anomalies into a central vortex core, and (ii) the efficient conversion of diabatic heating to rotational energy that drives the system-scale intensification. Although not explicitly stated, the importance of the low-deformation/enhanced-vorticity flow region for the above two TC formation mechanisms is implied in the first and third hypotheses of Dunkerton et al. (2009), respectively.

In this paper we construct a diagnostic that effectively measures the level of solid body rotation, in order to identify this low-deformation/enhanced-vorticity region at the centre of quasi-closed circulations. The advantage of this approach is that the diagnostic is considerably simpler to calculate and objectively measure than it is to diagnose the quasi-closed circulation from wave-relative streamlines. The disadvantage is that a local region of low-deformation vorticity (LDV) does not guarantee a quasi-closed circulation (e.g., consider a half-circle of solid-body rotation). It follows that in a breaking wave the enhanced LDV must occur over a large enough area for the streamlines to rotate through $360^{\circ}$. Also, enhanced LDV cannot diagnose recirculating flow, because it only describes the flow at some instant in time. However, if the enhanced LDV is sustained one can assume recirculation if fluid parcels have had enough time to complete a full rotation about the circulation centre. In reality convective regions contain a mix of low and high deformation flow on the scale 
of convective vortices. Smoothing of the wind field is necessary to identify LDV on the pouch scale.

On the other hand quasi-closed, recirculating flow does not guarantee favourable conditions for TC formation (e.g., a weak trough with zero through-flow may not contain sufficient vorticity to spawn a TC). It follows that the larger in horizontal extent and the greater the magnitude of an enhanced cyclonic LDV anomaly the greater the dynamic potential for TC formation. We introduce a quantity based on the Okubo-Weiss ${ }^{2}$ parameter and absolute vorticity that measures LDV, which we label OWZ. We argue that OWZ is a simple diagnostic that can be used to identify vorticity rich quasi-closed circulations and hypothesise that All TC precursors contain enhanced OWZ. We test the hypothesis by identifying regions of enhanced OWZ associated with observed TCs using ERA-interim reanalysis data.

It will also be necessary for the enhanced OWZ to span the low- to mid-troposphere so that the developing TC vortex column will remain intact. If the pouch is seen to not only protect a developing TC, but to also contain (i) the vorticity that is concentrated into the TC vortex, and (ii) the moist and well mixed air that minimises evaporation and downdraft potential, then the pouch alignment is only necessary where the system-scale flow is convergent, and where the capacity for evaporation is high (i.e., the low- to mid-troposphere).

We show in this paper that using OWZ to diagnose potential TC formation is an improvement on the vertical component of vorticity used in the many existing TC genesis parameters and predictors (e.g., Gray, 1979; Royer et al., 1998; Emanuel and Nolan, 2004; Camargo et al., 2007; Tippett et al., 2011; Menkes et al., 2011), because it pinpoints favourable regions within larger areas of enhanced vorticity. However, like these genesis parameters and predictors, thermodynamic conditions are also incorporated into the OWZ parameter (described later), which shows some promise as a TC formation diagnostic and TC detector for coarse resolution models, including global climate models.

The OWZ approach to TC detection differs from traditional detectors that try to identify directly circulations that resemble TCs. Instead it identifies circulations that are favourable for development. A TC is declared if these conditions are sustained for a minimum period of time. The OWZ approach to TC detection will be documented in two forthcoming papers.

\footnotetext{
${ }^{2}$ In studies of two-dimensional flows and two-dimensional turbulence, the common dynamic parameter designating the existence of a separate vortex (or rotationally dominated flow regions) is the Okubo-Weiss parameter (Okubo, 1970; Weiss, 1991). It is commonly used to designate the existence of "coherent" vortex structures (Haller and Yuan, 2000; Provenzale, 1999) including ocean eddies (e.g., Isern-Fontanet et al., 2006; Cruz-Gomez and Bulgakov, 2007). The Okubo-Weiss parameter has been used in studies of TC intensification (Rozoff et al., 2006) and TC genesis (Dunkerton et al., 2009).
}

The OWZ is derived and discussed in the next section. The data and detection and tracking methods used are described in Sect. 3. The core hypothesis that all TC precursors contain enhanced OWZ is tested in Sect. 4.1, and the development of an OWZ parameter that incorporates thermodynamic necessary conditions is presented in Sect. 4.2. Possible applications of the OWZ parameter are discussed in Sect. 5, and the paper is summarised in Sect. 6.

\section{Low deformation vorticity}

The outermost quasi-closed, wave relative streamline essentially defines the protective pouch of Dunkerton et al. (2009). Dunkerton et al. found that the TCs formed near the pouch centre where the trough-axis and the wave critical latitude intersect, which they labelled the TC formation sweet-spot. Because the calculation of the wave relative streamlines and the wave critical latitude both require knowledge of the wave phase speed, it can be difficult to identify and track the TC formation sweet-spot; the sweet-spot cannot be determined from instantaneous data. In this paper we propose a new, alternative dynamic quantity to identify and quantify the TC formation sweet-spot that is Galilean invariant and thus identifiable in instantaneous data.

Our new quantity is related, but distinct from, the familiar Galilean-invariant vorticity, which is used in most TC formation diagnostics, and indeed the vertical component ${ }^{3}$ is enhanced surrounding the sweet-spots identified by Dunkerton et al. and subsequent papers by these authors (Wang et al., 2009; Montgomery et al., 2010; Wang et al., 2010a, b; Wang, 2012; Wang et al., 2012, hereafter termed DMW papers). However, enhanced vorticity can often extend far from the sweet-spot, which means vorticity alone is insufficient for identifying the sweet-spot. In contrast, enhanced levels of the Okubo-Weiss parameter in the pouch are only found surrounding the sweet-spot in the DMW papers. Indeed the Okubo-Weiss parameter was used by these authors to diagnose circulations with formation potential in near real time during the PREDICT experiment (Montgomery et al., 2012).

The Okubo-Weiss parameter (OW) is a measure of the relative amount of vorticity to deformation flow,

$\mathrm{OW}=\zeta^{2}-\left(E^{2}+F^{2}\right)$.

Here $\zeta$ is the vertical component of relative vorticity, $E$ is the stretching deformation and $F$ is the shearing deformation. These quantities in Cartesian and cylindrical coordinates (Wang, 2008) are, respectively,

$\zeta=\left[\frac{\partial v}{\partial x}-\frac{\partial u}{\partial y}\right]=\left[\frac{\partial V}{\partial r}_{\mathrm{SV}}+\underline{V}_{\mathrm{CV}}-\frac{1}{r} \frac{\partial U}{\partial \lambda}\right]$

\footnotetext{
${ }^{3}$ Hereafter, our use of "vorticity" refers to the vertical component.
} 
$E=\left[\frac{\partial u}{\partial x}-\frac{\partial v}{\partial y}\right]=\left[\frac{\partial U}{\partial r}-\frac{U}{r}-\frac{1}{r} \frac{\partial V}{\partial \lambda}\right]$

$F=\left[\frac{\partial v}{\partial x}+\frac{\partial u}{\partial y}\right]=\left[\frac{\partial V}{\partial r}-\frac{V}{r}+\frac{1}{r} \frac{\partial U}{\partial \lambda}\right]$,

$u$ and $v$ are the zonal and meridional wind components, $x$ and $y$ are the zonal and meridional coordinate directions, $U$ and $V$ are the radial and tangential wind components, and $r$ and $\lambda$ are the radial and azimuthal coordinates.

From Eq. (2) it can be seen that OW is also Galilean invariant, (e.g., all terms in the Cartesian coordinate form are functions of wind gradients alone). However, the radius of curvature, and thus the shear and curvature vorticity are not Galilean invariant (Dunkerton et al., 2009). OW has greater potential for identifying TC formation locations than vorticity, because OW effectively distinguishes between vorticity with high flow curvature (a distinguishing feature of circular flow) and vorticity dominated by shear.

It can be useful to consider vorticity in cylindrical coordinates for axisymmetric flow to take advantage of the ability to separate vorticity into curvature and shear components (e.g., Holton, 2004). Holton's $\zeta$ in natural coordinates is essentially $\zeta$ in cylindrical coordinates when $U=0$. Thus for such flows the first and second cylindrical terms on the RHS of Eq. (2a) are equivalent to Holton's shear vorticity (SV) and curvature vorticity (CV) components respectively (see subscripts in Eq. 2a), and the stretching deformation $E$ is zero. In this framework parallel flow corresponds to an infinite radius of curvature, and the vorticity reduces to pure shear vorticity. In such flows $\zeta$ and $F$ are identical (cf. Eqs. 2a and 2c) and from Eq. (1) OW is zero.

For simplicity we consider horizontal axisymmetric flow, in which the radial flow is small so that streamlines are approximately circular. From Eq. (2c), zero deformation implies the shear vorticity is identical to the curvature vorticity, and solid body vorticity (SBV) reduces to, $\zeta_{\mathrm{SBV}}=\frac{2 \mathrm{~V}}{r}$. Because the developing TC system vorticity tendency is greater in an environment of enhanced absolute vorticity ${ }^{4}$ $(\eta=\zeta+f)$ and because the balanced vortex intensification becomes more efficient (for a given heat source) with increasing inertial stability ${ }^{5}(I)$, the most favourable location for TC formation will be a finite region within the pouch where both $\eta$ and $I$ are largest. The inertial stability squared is given by,

$I^{2}=(\zeta+f)\left(\frac{2 V}{r}+f\right)$.

\footnotetext{
${ }^{4}$ The dominant $\eta$-tendency term is equal to the horizontal inward flux of $\eta$.

${ }^{5}$ Solutions to the Sawyer Eliassen equations demonstrate greater efficiency in converting potential energy to kinetic energy with increasing $I$ (e.g., Shapiro and Willoughby, 1982; Schubert and Hack, 1982; Hack and Schubert, 1986).
}

To identify the most favourable formation region in our simplified axisymmetric pouch, we consider the streamline that contains the highest average vorticity and inertial stability. From Stokes' circulation theorem the area-integrated vorticity inside a circular streamline of radius $r$ is given by,

$\iint \zeta \mathrm{d} r \mathrm{~d} \lambda=2 \pi r V$

Dividing by the area of the closed streamline gives the areaaveraged vorticity, or vorticity concentration, within that streamline. Thus,

$\bar{\zeta}(r)=\frac{2 V}{r}=2 \omega_{r}$,

where $\omega_{r}$ is the angular frequency at radius $r$, and the overbar represents the area average. It follows that the streamline containing the highest concentration of vorticity is the streamline with maximum $V / r$ or maximum angular frequency. A similar argument can be used to show that the area-averaged $V / r$ and thus $I^{2}$ (Eq. 3) is greatest inside the maximum $V / r$ streamline for solid body rotation (i.e., when $\omega$ is constant inside that stream line).

A plot of $V$ as a function of $r$ for an axisymmetric circulation is depicted in Fig. 1 (blue curve). It shows $V$ increasing with $r$ up until the radius of maximum wind (RMW) and decreasing beyond that. Any straight line of positive gradient passing through the origin and intersecting the curve has a gradient equal to $V / r$ at the point (or points) of intersection. The gradient of the constant $V / r$ line that is tangent to the $V$-curve is equal to the maximum $V / r$ (Fig. 1, straight sloping purple line). Because this line touches at a tangent to the $V$-curve, $\frac{\partial V}{\partial r}=\frac{V}{r}$ at the intersection, which as noted above, describes solid body rotation. However, in Fig. 1 the region of $\frac{\partial V}{\partial r}=\frac{V}{r}$ (at $r=a$ where $V / r$ is a maximum) is infinitessimally small and essentially describes local solid body rotation. If a finite area of solid body rotation ( $\omega=$ constant) were present, (e.g., the blue curve exactly following the thick purple curve for some distance inward of $r=a$ ) then from Eq. (5) the area-averaged vorticity would be identical inside every streamline passing through that constant $\omega$ radius range. In that case, due to the larger enclosed area, we consider the outermost of these streamlines to contain the most favourable formation region. To generalise, the outermost streamline containing the maximum area-averaged vorticity occurs at the radius where the $V$-curve gradient first becomes less than the line of maximum $V / r_{\max }(r=a$, Fig. 1). Additionally, the maximum area- averaged inertial stability inside this streamline occurs for solid body rotation; where the blue curve $V$ exactly follows the thick purple line inside $r=a$. Thus, the most favourable region for TC formation resides inside the largest radius of maximum $V / r$ (shaded green in Fig. 1), and the flow is most favourable inside this region if it is in solid body rotation. In reality the formation environment is likely to have an annular structure similar to the blue curve in Fig. 1, although deviations from the 


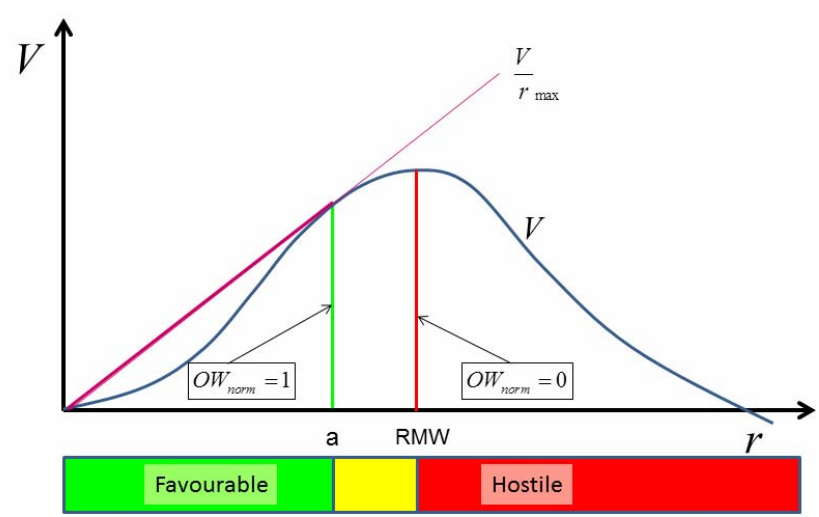

Fig. 1. Schematic representation of a hypothetical tangential wind $(V)$ profile (blue curve) with radius $(r)$. The green line at $r=a$ depicts the radius of maximum $\frac{V}{r}$, which is defined by the slope of the purple curve. At $r=a, \frac{V}{r}=\frac{\partial V}{\partial r}$, the flow is in solid body rotation and $\mathrm{OW}_{\text {norm }}=1$. Inside $r=a$ the area-averaged vorticity is maximized, which is deemed to be the most favourable region for TC formation. The red line depicts the radius of maximum wind (RMW) where $\mathrm{OW}_{\text {norm }}=0$, beyond which deformation exceeds vorticity and the environment is deemed to be hostile to TC formation.

thick purple line (and thus deviations from maximum areaaveraged $I^{2}$ ) are likely to be small, because vortices with $\frac{\partial V}{\partial r}$ increasingly greater than $\frac{V}{r}$ are more likely to be barotropically unstable.

For diagnosing favourable formation regions in rectangular gridded data it would be useful to have a Cartesian coordinate diagnostic that identifies maximum $V / r$. The maximum OW cannot be used because, for flows such as that depicted in Fig. 1, the maximum OW will be located at some radius less than $r=a$ where $\frac{\partial V}{\partial r}>\frac{V}{r}$. This relationship becomes apparent when the shear vorticity is expressed as a deviation $(\delta)$ from solid body rotation, i.e.,

$$
\frac{\partial V}{\partial r}=\frac{V}{r}+\delta
$$

Then OW reduces to,

$\mathrm{OW}=4 \frac{V}{r} \frac{\partial V}{\partial r}=4 \frac{V}{r}\left(\frac{V}{r}+\delta\right)$.

As noted above $\frac{\partial V}{\partial r}=\frac{V}{r}$ at the maximum $V / r$, which corresponds to $\delta=0$ in Eq. (7). Clearly, if $\delta>0$, OW exceeds the local solid body rotation, $\mathrm{OW}_{\mathrm{SB}}=4(V / r)^{2}$, and can conceivably exceed $\mathrm{OW}$ at the maximum $V / r(r=a)$. If on the other hand OW is normalised by the relative vorticity squared,

$\mathrm{OW}_{\text {norm }}=\frac{\zeta^{2}-\left(E^{2}+F^{2}\right)}{\zeta^{2}}$,

then using Eqs. (2a) and (6),

$\mathrm{OW}_{\text {norm }}=\frac{\mathrm{OW}}{\mathrm{OW}+\delta^{2}}$, which shows that $\mathrm{OW}_{\text {norm }}$ is maximized for solid body rotation $(\delta=0)$. It follows that $\mathrm{OW}_{\text {norm }}=1$ at the maximum $V / r$ and it must be less than 1 elsewhere. These differences between $\mathrm{OW}$ and $\mathrm{OW}_{\text {norm }}$ are illustrated in Fig. 2 for a wind profile similar to that of Fig. 1, although the square root of OW is plotted instead, so that it can be compared with relative vorticity $\zeta$, the $\zeta$ components, shear and curvature vorticity, and the OWZ parameter introduced below.

Figure 2 shows that for a $\delta>0$ wind profile $\left(\frac{\partial V}{\partial r}>\frac{V}{r}\right)$ OW can be maximised inside $r=a$, unlike $\mathrm{OW}_{\text {norm }}$ which is maximized at $r=a$. Figure 2 also shows both $\mathrm{OW}$ and $\mathrm{OW}_{\text {norm }}$ reduce to zero at the RMW, unlike $\zeta$ which maintains a positive contribution from the curvature vorticity term $V / r$.

Outside the $r<=a$ favourable region the formation potential decreases with increasing radius until the environment becomes hostile to formation. Where exactly the environment becomes hostile is not obvious. But it would seem unlikely that formation will occur where the deformation exceeds the vorticity $(\mathrm{OW}<0)$. In such environments developing vorticity anomalies are likely to be sheared apart (e.g., Rozoff et al., 2006). For a simplified axisymmetric, nondivergent environment, Eq. (7) demonstrates that the flow would become hostile to formation where $\frac{\partial V}{\partial r}<0$ (just outside the radius of maximum wind, shaded red in Fig. 1), and as $r \rightarrow \infty$ (parallel flow) $^{6}$. The normalized OW is also zero where $\mathrm{OW}=0$, which means the two extremes of $\mathrm{OW}_{\text {norm }}=$ 1 and 0 describe our formation favourability range of most favourable to hostile respectively (shaded yellow in Fig. 1). Because $\mathrm{OW}_{\text {norm }}$ is Galilean invariant in Cartesian coordinates and is simple to calculate in non-axisymmetric flows, it makes an ideal TC formation favourability scale parameter, assuming the above TC formation favourability arguments are approximately valid for the non-axisymmetric, but still highly curved flows of typical TC formation regions.

Due to its long-recognised importance for TC formation we choose to weight the absolute vorticity $(\eta)$ with positive values of $\mathrm{OW}_{\text {norm }}$ to get a quantity that reflects the solid body component of cyclonic absolute vorticity. We label the quantity OWZ, in recognition of the dominant contributions of the OW parameter and the vertical component of relative vorticity $(\zeta)$ to the quantity, i.e.,

$\mathrm{OWZ}=\max \left(\mathrm{OW}_{\text {norm }}, 0\right) \times \eta \times \operatorname{sign}(f)$.

Here the parameter is multiplied by the sign of $f$ to ensure OWZ is positive and negative for cyclonic and anticyclonic flow curvature respectively in both hemispheres. It is clear from Eq. (10) that OWZ has the same magnitude as $\eta$ for solid body rotation, and is zero for flows with zero curvature vorticity or flows in which the deformation exceeds the vorticity. A comparison of OWZ with OW can be made using

\footnotetext{
${ }^{6}$ We ignore $V=0$ here because apart from the origin, it reaches zero in our simplified example at a radius larger than the radius of maximum wind.
} 


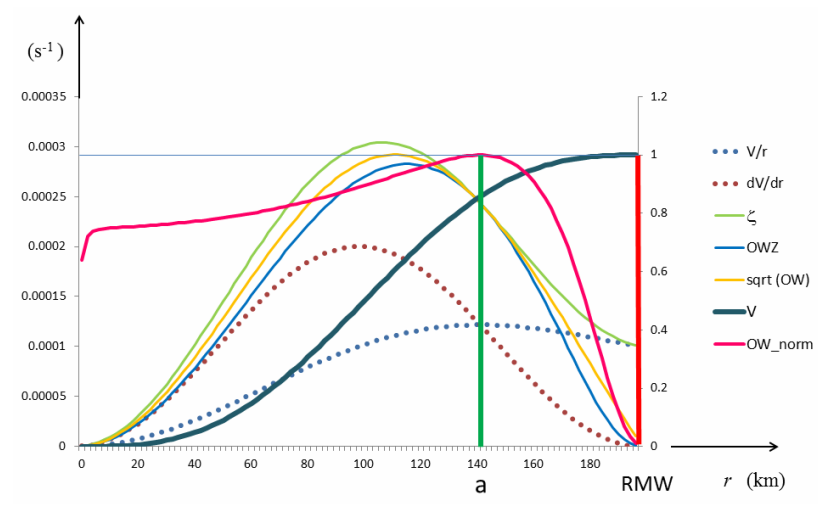

Fig. 2. Hypothetical tangential wind (thick blue curve) similar to that shown in Fig. 1 from $r=0$ to the RMW at $200 \mathrm{~km}$, with associated vorticity terms: relative vorticity (thin green curve), curvature vorticity (blue dotted curve), shear vorticity (brown dotted curve), the square root of the Okubo-Weiss parameter (thin yellow curve), the normalised Okubo-Weiss (mauve curve), and zero Coriolis OWZ (pale blue curve). The vorticity scale is given by the left vertical axis, and the $\mathrm{OW}_{\text {norm }}$ scale is given in the right vertical axis. The maximum tangential wind is $20 \mathrm{~ms}^{-1}$. The green and red vertical lines are included for ease of comparison with the Favourable and Hostile regions of Fig. 1. The blue horizontal line represents $\mathrm{OW}_{\text {norm }}=1$.

Fig. 2. For a meaningful comparison the square root of $\mathrm{OW}$ is plotted alongside OWZ with zero Coriolis. The figure shows very little difference between the two curves, and indeed for smaller $\delta$ the differences reduce even further. Thus the main difference between the two parameters is the coriolis component in OWZ, which turns out to be very important when used as a genesis parameter ${ }^{7}$.

\section{Data and detection method}

The detection, tracking and verification of TC-like circulations is a multi-step process, in which circulations with the dynamic potential to develop into a TC are first identified and tracked. In order to study the nature of the circulations along the storm tracks, the circulations are then tested by applying specific conditions and thresholds, which the storm track either passes or fails. Ultimately these conditions and thresholds are tuned such that a pass represents a TC detection. Unlike traditional TC detectors there is no test for the existence of a TC-like circulation. Instead the detector tests for conditions favourable for TC formation. If these conditions are sustained for a sufficient period of time a TC is declared. (A mature TC will also satisfy these conditions.) In the ver-

\footnotetext{
${ }^{7}$ Experiments were performed with OWZ replaced by $\sqrt{\mathrm{OW}}$ in the prediction system. When the $\sqrt{\mathrm{OW}}$ thresholds were tuned to give the best overall performance, the false alarm rate within $10^{\circ}$ of the equator was 11 times the miss rate, compared with a near oneto-one OWZ false alarm/miss ratio at latitudes greater than $10^{\circ}$.
}

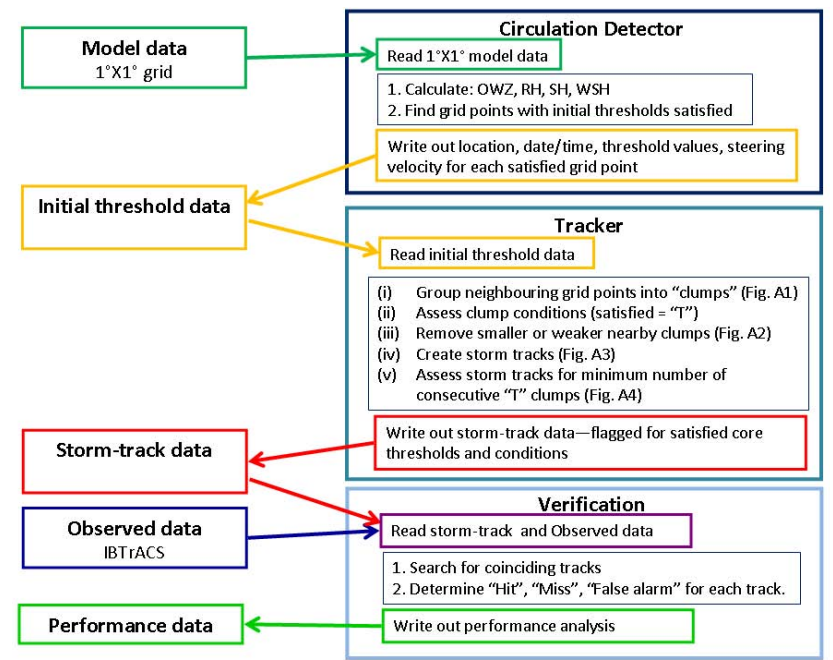

Fig. 3. Schematic representation of the detection, tracking and verification procedures used in the OWZP detection system. The five steps in the Tracker box are further illustrated in Figs. A1-A4.

ification process the storm tracks that both pass and fail are compared with observed TC tracks. A schematic illustration of this multi-step process is provided in Fig. 3.

\subsection{Data}

In order to test the hypothesis that enhanced OWZ is a necessary precursor to TC formation, globally consistent datasets are required. Global reanalysis data provide a level of consistency; however differing availability of observations used in the reanalysis will contribute to unavoidable inconsistencies from basin to basin, and from event to event. A consistent TC database is also important for determining which circulations should be considered TCs. Due to a degree of subjectivity in determining circulation intensity from remote sensing (Dvorak technique) inconsistencies have been reported in the only global TC database available: IBTrACS (Knapp et al., 2009). With these inconsistencies there will be a degree of unavoidable uncertainty with the chosen OWZ threshold values. In order to reduce inconsistencies as much as possible we have used a universal TC definition of $17 \mathrm{~ms}^{-1}$ sustained (10 min mean) wind. The IBTrACS data set incorporates cyclone best-track data from all global forecast offices and warning centres. For those cyclones tracked by more than one warning centre there are alternative versions of the track and TC intensity. In such cases we use the track and intensity measure from the forecast centre with WMO responsibility for that TC basin. Various warning centres designate the best track with different metrics for the TC intensity, including central pressure, $1 \mathrm{~min}$ average sustained winds and $10 \mathrm{~min}$ average sustained wind. The analyses in the current study used the conversion factor of 0.871 (Knapp and Kruk, 2010) to convert from $1 \mathrm{~min}$ sustained winds to $10 \mathrm{~min}$ sustained 
winds. While Harper et al. (2010) have suggested a more appropriate conversion factor of 0.93 , we note that the error near $17 \mathrm{~ms}^{-1}$ is minimal and falls within the uncertainty associated with the reporting of wind speeds in 5 knot increments.

The scale of precursor disturbances within which TCs form is typically of the order of hundreds of $\mathrm{km}$. McBride and Zehr's (1981) composite studies identified enhanced values of relative vorticity on a scale of about $1200 \mathrm{~km}$, which is consistent with the individual TC precursors found by Davidson et al. (1990) in the Australian Monsoon experiment. These precursors are resolvable in relatively coarse resolution model data, including the ERA-interim reanalysis data (at a horizontal resolution of $1.5^{\circ} \times 1.5^{\circ}$, Dee et al., 2011). At the time of development a future goal was to use the OWZ parameter as a TC detector in climate model data, so a decision was made when developing the OWZ parameter to use the ERA-interim re-analysis data $24 \mathrm{~h}$ apart (00:00 UTC). In order to minimise the effects of varying resolution between climate models, it was anticipated that interpolation to a common grid would be important. A grid of $1^{\circ} \times 1^{\circ}$ was chosen.

\subsection{Initial thresholds}

The purpose of the initial thresholds is to identify circulations that have the dynamic potential to support TC formation. The initial OWZ thresholds were chosen after contouring OWZ for a number of events in the South Pacific and Western-North Pacific to get a feel for the OWZ values in the $48 \mathrm{~h}$ prior to TC declaration. This led to the development of an initial OWZ parameter (hereafter OWZP) with thresholds of 50 and $40 \times 10^{-6} \mathrm{~s}^{-1}$ on the 850 and $500 \mathrm{hPa}$ pressure levels respectively. Additional, relatively weak thermodynamic and dynamic thresholds were added to the initial OWZP to eliminate any obviously non-tropical flows, while being careful to avoid eliminating exceptional cases with potential for development. These relatively weak thresholds are relative humidity greater than $70 \%$ and $50 \%$ on the 950 and $700 \mathrm{hPa}$ pressure levels respectively, and an 850 to $200 \mathrm{hPa}$ vertical wind shear threshold of $25 \mathrm{~ms}^{-1}$.

\subsection{Circulation detector}

The circulation detection routine takes reanalysis or climate model data interpolated to $1^{\circ} \times 1^{\circ}$ grid, and calculates OWZ on 850 and $500 \mathrm{hPa}$ levels, relative humidity on 950 and $700 \mathrm{hPa}$ levels, specific humidity on the $950 \mathrm{hPa}$ level, the 850 to $200 \mathrm{hPa}$ vertical wind shear and a $700 \mathrm{hPa}$ steering velocity for each model grid point on those levels. The steering velocity used is the average $700 \mathrm{hPa}$ wind within a $4^{\circ} \times 4^{\circ}$ box centred on the grid point. Other levels and combinations of levels were tested, as was the size of the averaging box, with the above producing the best result. The wind shear was calculated from smoothed wind data to: (i) better represent the broader shear environment, and (ii) avoid the storm-system shear exceeding the threshold as storms intensified. The spatial smoothing involved three applications of a weighted average, with the grid point at the centre of the smoothing receiving a weighting of $1 / 4$, and the immediate neighbours (north, south, east and west) and the diagonal neighbours receiving a weighting of $1 / 8$ and $1 / 16$ respectively. The wind shear is calculated as the vector difference between the 850 and $200 \mathrm{hPa}$ smoothed winds.

At each grid point where the above initial thresholds are satisfied, the circulation detector outputs latitude, longitude, date, time, the threshold values, plus the wind components of the steering velocity. All other information is discarded.

\subsection{Tracker and TC detection}

The tracker is described here briefly with more detail in Appendix A. It reads output from the circulation detector and performs the following steps:

i. neighbouring groups of detected grid points are grouped together to create a single "clump" of grid points,

ii. each clump is assessed to see if it satisfies a set of clump conditions,

iii. clumps in close proximity are reduced to one clump by discarding the weaker or smaller clumps,

iv. storm tracks are constructed by stringing together clumps from consecutive times,

v. storm tracks are assessed to see whether they satisfy an additional track condition of minimum number of consecutive clumps satisfying the clump conditions.

While the initial thresholds are used to identify circulations worth tracking, clump conditions are used to test the state of the circulations along the track. The clump and track conditions can be set to investigate OWZP sensitivity, or to objectively determine TCs.

The clump requirements include: a minimum size limit, and a land-impact condition. In all experiments discussed and presented in this paper the minimum size limit is set to two neighbouring grid points, and the land condition is applied only to the last four experiments labelled OWZP2-5 in Table 1. In the experiments presented in this paper, the track condition is varied between one and three minimum consecutive clumps satisfying the clump conditions (labelled "Minimum consecutive "T" clumps" in Table 1). The satisfied track condition is a culmination of all conditions being satisfied, which represents a TC detection in the final experiment (OWZP5, Table 1). Thus, the TC detector itself is contained in the Tracker algorithm.

\subsection{Track verification}

In order to investigate the core hypothesis that enhanced OWZ is necessary for TC formation, analysed tracks are 
Table 1. Core thresholds and track condition for the various OWZ parameter (OWZP) experiments, with the associated "hit" and "false alarm" rates in the last two columns. The "Hit" rate represents the percentage of observed TC tracks with a matching "detected" track that has all clump conditions satisfied for a minimum number of consecutive times. The "False Alarm" rate represents a ratio of false detections to observed TCs expressed as a percentage. Subscripts in the threshold labels refer to pressure levels. $\mathrm{RH}=$ relative humidity, $\mathrm{SH}=\mathrm{specific}$ humidity and Wsh = wind shear. Threshold values in bold are "Non-limiting", i.e., they are nearly always satisfied in wet-season, oceanic, tropical convection. Approximate false alarm rates are given in italics for the OWZ necessity testing experiments, in which the false alarm rate is irrelevant.

\begin{tabular}{|c|c|c|c|c|c|c|c|c|c|}
\hline & $\begin{array}{r}\mathrm{OWZ}_{850} \\
\left(\times 10^{-6} \mathrm{~s}^{-1}\right)\end{array}$ & $\begin{array}{r}\mathrm{OWZ}_{500} \\
\left(\times 10^{-6} \mathrm{~s}^{-1}\right)\end{array}$ & $\begin{array}{r}\mathrm{RH}_{950} \\
(\%)\end{array}$ & $\begin{array}{r}\mathrm{RH}_{700} \\
(\%)\end{array}$ & $\begin{array}{r}\mathrm{Wsh}_{850-200} \\
\left(\mathrm{~ms}^{-1}\right)\end{array}$ & $\begin{array}{r}\mathrm{SH}_{950} \\
\left(\mathrm{~g} \mathrm{~kg}^{-1}\right)\end{array}$ & $\begin{array}{l}\text { Minimum } \\
\text { consecutive } \\
\text { "T" clumps }\end{array}$ & $\begin{array}{l}\text { Hit } \\
(\%)\end{array}$ & $\begin{array}{r}\text { False Alarm } \\
(\%)\end{array}$ \\
\hline OWZP1 & 50 & 40 & 70 & 50 & 25 & 10 & 1 & 95.9 & $>1000$ \\
\hline OWZP Hi & 60 & 50 & 70 & 50 & 25 & 10 & 1 & 93.0 & $>1000$ \\
\hline OWZP Lo & 40 & 30 & 70 & 50 & 25 & 10 & 1 & 96.1 & $>1000$ \\
\hline OWZP2 & 50 & 40 & 85 & 70 & 12.5 & 10 & 1 & 94.5 & $\sim 450$ \\
\hline OWZP3 & 50 & 40 & 85 & 70 & 12.5 & 10 & 3 & 85.3 & 55 \\
\hline OWZP4 & 50 & 40 & 85 & 70 & 12.5 & 12.3 & 3 & 85.0 & 48.4 \\
\hline OWZP5 & 60 & 50 & 85 & 70 & 12.5 & 12.3 & 3 & 78.0 & 25.7 \\
\hline
\end{tabular}

compared with observed TC tracks. A simple track verification scheme was devised in which each clump position within the analysed track is compared with the observed positions of TC tracks in the IBTrACS database. If at least one match of position and time is made within a $2.5^{\circ}$ tolerance, the analysed track is deemed to match the observed track. (Tolerances ranging from $1^{\circ}$ to $4^{\circ}$ were tested, with $2.5^{\circ}$ yielding the best result.) With the TC detector application in mind, each match is labelled a "hit", each un-matched analysed track is considered to be a "false-alarm", and each un-matched observed track is considered to be a "miss". To ensure only systems in the IBTrACS database that reached our chosen TC definition were considered, only that part of the observed track greater than $17 \mathrm{~ms}^{-1}$ was included in the comparison.

The tracker and track verification are also used to investigate the relationship between observed TCs and enhanced OWZ (and other thresholds) diagnosed in the ERA-interim reanalysis data. A study of the hit, miss and false alarm rates, while systematically adjusting thresholds and other conditions, enables an assessment of the necessity of these conditions for TC formation. This is the primary focus of Sect. 4 . It is important to note that when the track verification is applied in this way, a "hit" does not necessarily represent a TC, instead it indicates that the condition being tested for is positive. The tracker with conditions and thresholds set for TC detection is discussed in Sect. 5.2.

\section{A new approach to necessary conditions for TC formation}

At the centre of this investigation is the question of whether low-deformation recirculating flow near the centre of a quasiclosed circulation is necessary for TC formation. In the absence of a simple method for identifying system-relative quasi-closed circulations the enhanced values of OWZ is introduced as a low-deformation vorticity proxy due to its theoretical association with recirculating flow likely to support system-scale, approximately balanced, vortex intensification, discussed in Sect. 2.

If a condition is met in every TC formation environment, it could be argued that the condition is "necessary" by association for TC formation. Testing for this condition would yield a $100 \%$ hit $(0 \%$ miss $)$ rate if the observational data were perfect. The necessary condition may also be present in non-developing environments. Thus the "false alarm" rate is irrelevant for "necessity" testing. The false alarm rate only becomes important when considering sufficiency. A condition or set of conditions is considered to be "sufficient" for TC formation when it accurately distinguishes between developing and non-developing TCs. Thus the false alarm rate can be used in combination with the hit (or miss) rate to measure the degree of sufficiency.

While recognising no observational or reanalysis data-set is perfect, we ask the following questions: (i) what percentage of observed TCs is associated with enhanced OWZ, (ii) is the percentage high enough to consider enhanced OWZ to be necessary for TC formation, and if so (iii) is this necessary condition more useful than the long recognised necessary condition of enhanced absolute vorticity? We address questions (i) and (ii) in Sect. 4.1, where we suggest enhanced OWZ is most-likely necessary for TC formation, but leave question (iii) to Sect. 5.1 where direct comparisons between OWZ and absolute vorticity are illustrated graphically.

While enhanced OWZ may be necessary for TC formation, OWZ alone, like vorticity in other TC formation parameters, is far from sufficient as a TC predictor. In Sect. 4.1 we attempt to establish the "necessity" of enhanced OWZ, and thus focus on the hit rate. In Sect. 4.2 we document the construction of the OWZ parameter that we later use as a TC 
detector, and demonstrate the increasing sufficiency of the OWZP (by a falling false alarm rate) as more realistic thermodynamic and wind shear thresholds are applied.

\subsection{Low deformation vorticity: a necessary condition for TC formation}

The central hypothesis of this paper is that all TCs form in a region of enhanced OWZ that spans the low- to mid-troposphere. Raymond and López Carrillo (2011) have shown that vertical alignment of the pouch is not critical, but suggests there should be some overlap. This result is consistent with our experience, using data interpolated to a $1^{\circ} \times 1^{\circ}$ grid, that an overlap which encompasses at least 2 neighbouring grid points is generally required. Thus in all experiments and tests we require all thresholds to be satisfied at a minimum of two neighbouring grid points. Compared with the partial pouch alignment condition of Raymond and López Carrillo (2011), our condition of overlapping enhanced OWZ is a stricter requirement, because it requires overlapping central pouch regions ${ }^{8}$.

Even if the hypothesis is true we would not expect the above OWZP to be satisfied in the reanalysis data for every TC in the IBTrACS database, due to imperfect data, subjective TC declarations, and coarse resolution reanalysis modelling. Using the somewhat arbitrarily chosen initial thresholds of $850 \mathrm{hPa} \mathrm{OWZ} \geq 50.0 \times 10^{-6} \mathrm{~s}^{-1}$ and $500 \mathrm{hPa} \mathrm{OWZ} \geq$ $40.0 \times 10^{-6} \mathrm{~s}^{-1}$ circulations were identified and tracked. As mentioned in Sect. 3.2 additional non-OWZ initial thresholds were applied to reduce the number of obvious, non-tropical circulations being identified. These are the $950 \mathrm{hPa}$ relative humidity $(\mathrm{RH}) \geq 70.0 \%, 700 \mathrm{hPa} \mathrm{RH} \geq 50.0 \%$, and 850 $200 \mathrm{hPa}$ vertical wind shear $\leq 25 \mathrm{~ms}^{-1}$. The initial thresholds determine the circulations to be tracked, while the core thresholds are imposed to decide whether the circulations satisfy a set condition at some time in the track history. These latter core thresholds and additional conditions comprise the OWZP. In this subsection we discuss the performance of the first OWZP (labelled OWZP1 in Table1) which uses the above initial thresholds plus one core threshold ( $950 \mathrm{hPa}$ specific humidity $\geq 10.0 \mathrm{~g} \mathrm{~kg}^{-1}$ ) to further eliminate non-tropical circulations. Of the 1574 TCs in the database ${ }^{9} 95.9 \%$ satisfied this criterion for at least one 00:00 UTC time period. An inspection of the remaining $4.1 \%$ of TCs showed that $1.8 \%$ had strong OWZ signatures at either 500 or $850 \mathrm{hPa}$ but not both, or on both levels but not sufficiently overlapping, $0.3 \%$ were missed because they

\footnotetext{
${ }^{8}$ The pouches of DMW include the high deformation $(\mathrm{OW}<0)$ regions surrounding the formation sweet-spot that protect the central region of low-deformation vorticity. The TC development occurs inside this protective layer, which in Fig. 1 extends well into the "Hostile" range.

${ }^{9}$ This includes all circulations that satisfied our condition of windspeed exceeding $17 \mathrm{~ms}^{-1}$ at one or more 00:00 UTC time, during the $20 \mathrm{yr}$ period $1989-2008$.
}

were too close to another circulation, $0.5 \%$ were missed due to the tracking algorithm merging the circulation with another event (i.e., the tracker did not recognise that there were two events), $1.1 \%$ were very short lived and perhaps borderline TCs (they had only one 00:00 UTC entry in the observed database), and there was no obvious reason for the remaining $0.4 \%$ of misses.

This low miss rate is not very sensitive to the choice of OWZ thresholds used (discussed below). It could be argued that with the current system design the misses due to storms in close proximity, the false merging of tracks, and the very short lived storms, are essentially unavoidable. Subtracting these "unavoidable" misses reduces the miss rate to $2.2 \%$, which suggests the hypothesis that enhanced OWZ in the low- to mid-troposphere is necessary for TC formation is well supported. However, $4.9 \%$ of the circulations with a matching TC in the TC database first met the criteria at the time of declaration, and $1.5 \%$ met the criteria after the TC had been declared. These examples are obviously too late to be considered cases in which the satisfied OWZP criteria represent evidence of formation within a protected pouch (a mature TC will also satisfy the OWZP criteria). However, given that the data time interval is $24 \mathrm{~h}$, and given the uncertainty that often surrounds the timing of TC declarations, it would be expected that some fraction of these would have satisfied the OWZP criteria for a number of hours prior to TC formation. At present we are not aware of any study that reports on the minimum time it takes for a TC to form once a partially overlapping protected environment is in place.

Every genesis event is different and there is considerable variation in the time it takes for TCs to form. When conditions are slightly unfavourable it can take many days for a relatively strong circulation to develop to TC intensity. However, at present we are only considering the history of the OWZP as a pouch indicator and not other factors such as shear and relative humidity that may hinder formation. The OWZP1 criteria were satisfied for the majority of TCs $(89.5 \%)$ at least one time period $(24 \mathrm{~h})$ prior to the TC declaration. In $78.3 \%$ and $59.5 \%$ of cases the criteria were satisfied for at least 2 and 3 time periods, respectively, and in $37.4 \%$ of cases the criteria were satisfied for 4 or more days.

How sensitive is the choice of OWZ thresholds? It is important for the thresholds to be set at a sufficiently restrictive level to identify circulations with potential to develop while ignoring the small-scale, small amplitude low-deformation vorticity noise. It might be assumed that a $100 \%$ hit rate could be obtained by lowering the thresholds until a circulation (with at least some vertical alignment) was found to match each observed TC. However, for OWZ threshold values of approximately half those reported here, the numbers of identified circulations increased significantly, leading to considerable circulation noise that became difficult to track and these tracks became difficult to match with observed TCs. The sensitivity to numbers of missed TCs was tested by varying the thresholds by $\pm 10 \times 10^{-6} \mathrm{~s}^{-1}$ in increments of 
$5 \times 10^{-6} \mathrm{~s}^{-1}$. The two extreme experiments (both thresholds increased and reduced by $10 \times 10^{-6} \mathrm{~s}^{-1}$, labelled OWZP_Hi and OWZP_Lo, respectively in Table 1) showed only minimal variation in percentage of miss rates, with the relaxed thresholds producing a reduction in miss rate of less than $0.2 \%$, and the more restrictive threshold increasing the miss rate by $2.9 \%$ (Table 1 ). The thresholds are of similar order to the OWZ (i.e., $70 \times 10^{-6} \mathrm{~s}^{-1}$ ) we estimated by comparing the absolute vorticity and OW for TS Chris at $600 \mathrm{hPa}$ just prior to genesis, from of an early version of Dunkerton et al. (2009).

When optimising threshold values to maximise hits and minimise misses and false alarms, the more restrictive thresholds were found to be most ideal, as will be reported in a subsequent paper.

In summary, the above analysis shows that for about $90 \%$ of observed TCs, a circulation exists in the ERA-interim database in which OWZP1 is satisfied for $24 \mathrm{~h}$ or more prior to the time of declaration. Furthermore these numbers are not particularly sensitive to the choice of OWZ threshold, which shows the OWZP applied to ERA-interim reanalysis data can identify most circulations with the potential for TC formation. This result answers question (i) posed at the beginning of the section. However, question (ii) asks is this $90 \%$ value large enough for enhanced OWZ to be considered necessary for TC formation. More specifically, is enhanced OWZ on the two pressure levels in ERA-interim data corresponding to approximately $90 \%$ of observed TCs $24 \mathrm{~h}$ prior to declaration large enough to be considered necessary? If the remaining $10 \%$ can be written off due to imperfect data and detection technique, then one can conclude that it is quite likely to be necessary for TC formation. Regardless, the value of $90 \%$ demonstrates that OWZ can be a very useful quantity.

\subsection{Minimal wind shear and enhanced relative humidity: necessary conditions for TC formation}

Favourable conditions for TC formation have been recognised for decades (e.g., Gray, 1968). There is a broad consensus regarding the climatalogical conditions associated with TC formation (summarised in Tory and Frank, 2010), which can be split into thermodynamic and dynamic conditions. The dynamic conditions include enhanced low-level absolute vorticity and weak to moderate vertical wind shear. The former is incorporated in the $850 \mathrm{hPa}$ OWZ threshold. The thermodynamic conditions include (i) sea-surface temperatures in excess of $26.5-27.0^{\circ}$ coupled with a mixed layer ocean of about $50 \mathrm{~m}$ or greater, (ii) a deep surface-based layer of conditional instability, (iii) organised deep convection in an area of large-scale mean ascent and high mid-level relative humidity.

While these conditions have long been recognised as necessary for TC formation, they are by no means sufficient. Additionally, it is not entirely clear to what extent these conditions are necessary for TC development and how much they are symptoms of a developing TC. In this study we took a step back from the traditional necessary conditions and considered the fundamental dynamic and thermodynamic processes required to build a TC-scale vortex. Recognising that a large upward mass flux spanning the troposphere is required to converge sufficient vorticity to form the TC vortex, we considered the type of convection that can drive this mass flux, and the necessary thermodynamic conditions that allow such convection. Mapes and Houze $(1992,1993,1995)$ identified two dominant tropical precipitation types: deep convection, and stratiform. Deep convective regions are associated with upward mass flux spanning the troposphere fuelled by condensational heating, and stratiform precipitation regions are associated with lower troposphere downward mass flux fuelled by evaporative cooling, and upper troposphere upward mass flux. It follows that thermodynamic conditions of minimum evaporation potential (high humidity, moistneutral vertical profile, e.g., see the DCAPE discussion in Tory and Frank, 2010) are necessary to maximise the vertical mass flux. Conditions that favour broad regions of deep convection support increased vertical mass flux. Enhanced equivalent potential temperature in the mid-troposphere and above the surface layer was observed by Kingsmill and Houze (1999) to be associated with deep broad convection as distinct from deep narrow convection.

The thermodynamic theory and observations of the previous paragraph are consistent with the necessary thermodynamic conditions (ii) and (iii) above, and suggest that enhanced relative humidity at some level not far above the boundary layer and in the middle troposphere, could be used as an effective threshold for determining conditions favourable for TC formation. The sea surface temperature thermodynamic condition was disregarded, because it influences the rate at which heat and moisture can be transferred to the atmosphere, i.e., it does not provide a measure of the moisture currently in the atmosphere, which low-level relative humidity and specific humidity thresholds do provide. The outstanding dynamic necessary condition of weak to moderate vertical wind shear is addressed by imposing a more restrictive $850-200 \mathrm{hPa}$ vertical wind shear threshold than the $25 \mathrm{~ms}^{-1}$ of the initial threshold.

In the previous subsection the initial thresholds plus the core threshold of $950 \mathrm{hPa}$ specific humidity (OWZP1) applied to the ERA-interim reanalysis data led to the identification of about 11 circulations with the dynamic potential to develop for every circulation that matched a TC in the observed database. This result is not surprising because only the OWZ thresholds were set large enough to test for formation potential (the initial shear and moisture thresholds of OWZP1 are nearly always satisfied in the oceanic, wet-season tropics). Furthermore, we know that OWZ is not sufficient for identifying TC formation potential. By adding non-OWZ core thresholds that better match TC formation environments the ratio of detected circulations to observed TCs was significantly reduced. 
With the wind shear threshold reduced from 25 to $12.5 \mathrm{~ms}^{-1}$ (an often quoted shear threshold above which TC formation is unlikely, e.g., Zehr, 1992), and the RH thresholds increased to 85 and $70 \%$ on the 950 and $700 \mathrm{hPa}$ levels (OWZP2), about $23 \%$ of identified circulations matched TCs in the IBTrACS database, but at the cost of $22(1.4 \%)$ additional misses. The RH thresholds were relatively insensitive to variations of $\pm 5 \%$.

Assuming these core thresholds do capture the necessary thermodynamic and dynamic genesis ingredients, we still fall short of identifying sufficient formation conditions because the number of identified circulations that do not match TCs is quite high. However, as noted earlier the protective pouch studies highlight the importance of a sustained period of favourable conditions during which the proto-vortex gestates. With the condition that the initial plus core OWZ thresholds be met for at least three consecutive time periods (24 h apart, i.e., at least $48 \mathrm{~h}$, OWZP3) $61 \%$ of identified circulations can be linked to TCs in the observational database, but at the cost of $145(9.2 \%)$ additional misses.

These results show that about $85 \%$ of observed TCs were linked to a circulation in the ERA-interim database that satisfied the above dynamic and thermodynamic thresholds for three or more consecutive time periods. Furthermore, $73 \%$ and $56 \%$ of observed TCs were linked to ERA-interim circulations that had satisfied the above dynamic and thermodynamic thresholds for two and three 24-h time periods respectively at the time of TC declaration. This demonstrated increase in sufficiency shows that a further refined OWZP has the potential to be used as a TC genesis index. The extra conditions added to the OWZP effectively reduce the false alarm rate from in excess of $1000 \%$ to $55 \%$ and increased the miss rate to $15 \%$.

The main differences between an OWZ based index and traditional genesis indices are the time-scale over which the index is used for prediction, and the sufficiency. Traditional indices have been designed and used for seasonal timescales, and they provide an indication of the likelihood of TC formation in a relatively broad region. While these indices include conditions known to be necessary for TC formation, they are far from sufficient. However, because of the very transient nature of OWZ in tropical circulations, an OWZ based index would need to be confined to short term prediction and be specific to individual circulations. For that reason it has the potential to be more sufficient than traditional indices. If applied in this way the index takes on more of a TC detector role, because it is identifying conditions that are associated with imminent genesis.

In the next section we consider the potential application of an OWZ-based genesis parameter and we consider how well such a parameter might perform as a TC detector, and whether the imminent genesis qualities can be exploited in a TC formation diagnostic.

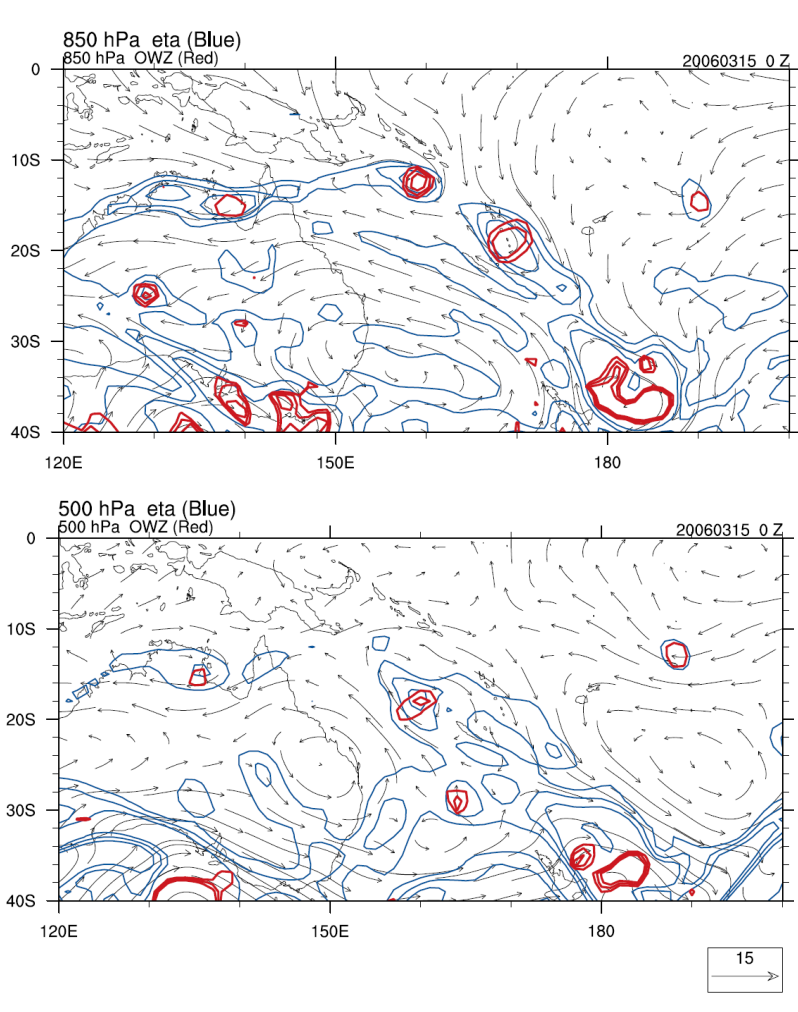

Fig. 4. ERA-interim absolute vorticity (blue contours, $-60,-80$, $-100 \times 10^{-6} \mathrm{~s}^{-1}$ ), OWZ (red contours, 60, 80, $100 \times 10^{-6} \mathrm{~s}^{-1}$ ) and horizontal winds (vectors) on $850 \mathrm{hPa}$ (upper panel) and $500 \mathrm{hPa}$ (lower panel) pressure levels, at 00:00 UTC 15 March 2006 ( $48 \mathrm{~h}$ prior to the formation of TC Larry). The TC Larry precursor is located at about $12^{\circ} \mathrm{S}, 160^{\circ} \mathrm{E}$.

\section{OWZP applications}

\subsection{Forecasting TC formation}

While enhanced low-level vorticity has long been recognised to be necessary for TC formation, enhanced OWZ pinpoints where the enhanced vorticity is likely to be associated with low deformation recirculating flow such as that surrounding the DMW sweet-spot, and is thus considerably more specific in identifying favourable environments for TC formation.

The absolute vorticity and OWZ can be compared in Fig. 4 for the south west Pacific $72 \mathrm{~h}$ prior to the formation of TC Larry (from ERA-interim reanalyses, 15 March 2006). The upper panel shows a near continuous band of enhanced cyclonic absolute vorticity at $850 \mathrm{hPa}$, of magnitude greater than $60 \times 10^{-6} \mathrm{~s}^{-1}$, in the monsoon trough stretching across northern Australia into the Coral Sea where it meets the South Pacific Convergence Zone (SPCZ), which runs approximately north-west to south east, which in turn meets a mid-latitude cyclone near $40^{\circ} \mathrm{S} 180^{\circ} \mathrm{E}$. Multiple vorticity centres are present in the tropical part of this band of lowpressure, but only three OWZ centres are present. The weakest OWZ centre in the Gulf of Carpentaria $\left(15^{\circ} \mathrm{S} 140^{\circ} \mathrm{E}\right)$ is 
only about half the magnitude of the absolute vorticity, which suggests shear vorticity is significant there. The TC Larry pre-cursor is located about $13^{\circ} \mathrm{S} 160^{\circ} \mathrm{E}$ and has a maximum OWZ magnitude similar to the absolute vorticity magnitude (both in excess of $100 \times 10^{-6} \mathrm{~s}^{-1}$ ), which suggests near solid body flow in the circulation centre. While the maximum absolute vorticity magnitude is similar in both examples, the latter is more likely to contain low deformation recirculating flow.

The lower panel of Fig. 4 shows absolute vorticity and OWZ on the $500 \mathrm{hPa}$ pressure level. The monsoon trough and SPCZ are not as strong as the $850 \mathrm{hPa}$ level, and the OWZ is also weaker and not in alignment with the OWZ centres below. Twenty four hours later, however, the 850 and $500 \mathrm{hPa}$ OWZ anomalies for both the TC Larry and TC Wati $\left(14^{\circ} \mathrm{S}\right.$ $190^{\circ} \mathrm{E}$ ) precursors are larger, more intense and aligned (not shown).

The monitoring of OWZ anomalies in the lower- and middle-troposphere provides important information on the evolution and vertical alignment of favourable formation circulations. After some limited monitoring of an experimental OWZ diagnostic during the previous northern Australian TC season, we found OWZ provided very useful insight into individual model behaviour. For example, in general a surface low will only intensify if there is alignment between the lowand mid-level OWZ anomalies. Shear can often separate the anomalies leading to a weakening of the surface low, while realignment will generally result in deepening of the surface low. Although it is beyond the scope of this paper to perform a detailed analysis of any TC formation event here, we highlight a few points of interest in the following OWZ analysis.

The OWZ evolution for a typical TC formation case is presented in Fig. 5 using reanalyses from the atmospheric component of the Australian Community Climate and Earth Systems Simulator (ACCESS, Puri et al., 2013), which covers the $96 \mathrm{~h}$ prior to the declaration of TC Zelia (Coral Sea, January 2011). (Note, the $850 \mathrm{hPa}$ OWZ is now contoured in blue, and the $500 \mathrm{hPa}$ OWZ contour intervals have been reduced compared to Fig. 4, to match the core thresholds of OWZP5 discussed in the next sub-section.) In the first panel the wind vectors show cyclonic shear vorticity on the south side of a westerly surge at $850 \mathrm{hPa}$ (between 12 and $15^{\circ} \mathrm{S}$, and 145 and $150^{\circ} \mathrm{E}$ ) with cyclonic curvature also evident in the wind vectors, consistent with the two OWZ maxima (blue contours). At $500 \mathrm{hPa}$ the OWZ maximum (red contours) is located between the two lower level maxima. Twelve hours earlier the $500 \mathrm{hPa}$ OWZ maximum (not shown) was aligned with the eastern $850 \mathrm{hPa}$ anomaly, with vertical wind shear most likely responsible for the separation. The westerly surge was particularly strong at this time, and the easterly $850 \mathrm{hPa}$ OWZ anomaly propagated about $3^{\circ}$ in the previous $12 \mathrm{~h}$ (not shown). In the first panel there is no sign of an earth relative closed circulation, which is not surprising given the strong westerly flow.
Twelve hours later (second panel) the $500 \mathrm{hPa}$ OWZ anomaly had intensified and become more aligned with the now broader $850 \mathrm{hPa}$ anomaly below. The westerly surge at $850 \mathrm{hPa}$ had also weakened, and easterly flow had appeared to the south, resulting in an apparent earth relative closed circulation. In the third panel the OWZ anomalies became more aligned and broadened, with the lower level anomaly intensifying in response to convective activity (not shown). In the fourth panel multiple OWZ centres appeared on both levels consistent with the outbreak of off-centre convection, which has reduced the symmetry and would be expected to slow the TC development. In the fifth panel the OWZ anomalies have become more symmetric again, and there is evidence of banding (consistent with vorticity filiamentation associated with the axisymmetrization process) in this and the next panel, particularly on the $500 \mathrm{hPa}$ level. In the seventh and eight panels the OWZ anomalies contract as the circulation intensifies, and there is considerable filiamentation of $850 \mathrm{hPa}$ OWZ in the eighth panel. In the last panel the contraction has continued and the two main OWZ anomalies are almost perfectly stacked vertically, apart from the filiamentation at $500 \mathrm{hPa}$. This is the time TC Zelia was declared a tropical cyclone.

Experimentation with the OWZP as a forecast tool has proved useful for understanding why numerical weather prediction models do or do not predict formation. An assessment of the MSLP alone is enough to identify whether an individual model is predicting formation, but it does not offer any indication of why. This can be confusing when multiple models are predicting multiple outcomes. In these situations the OWZP can be investigated to find out which "ingredient" is missing in a non-developing forecast. The robust requirement of sustained, overlapping OWZ in the low- to mid-troposphere provides a quick initial assessment of the development potential, which explains the majority of nondeveloping scenarios we observed. If the dynamic conditions are met for a non-developer, then an assessment of the thermodynamic conditions usually identifies dry air in the vicinity. By identifying the individual OWZP ingredients in each model, forecasters can make a more informed decision on accepting or rejecting the various model predictions.

\subsection{TC detection}

The relatively high percentage of TCs that have a corresponding circulation in the ERA-interim database in which the OWZP has been satisfied for three or more time periods ( $>48 \mathrm{~h}$ ) suggests that on the whole the OWZP does capture the necessary ingredients for genesis, and the requirement that the ingredients be present for a gestation period, perhaps brings us closer to the identification of sufficient conditions. If the OWZP method is reasonably successful in discriminating between developing and non-developing systems, then in theory it could be used as a TC detector in coarse resolution numerical model data. Having been developed in relatively 

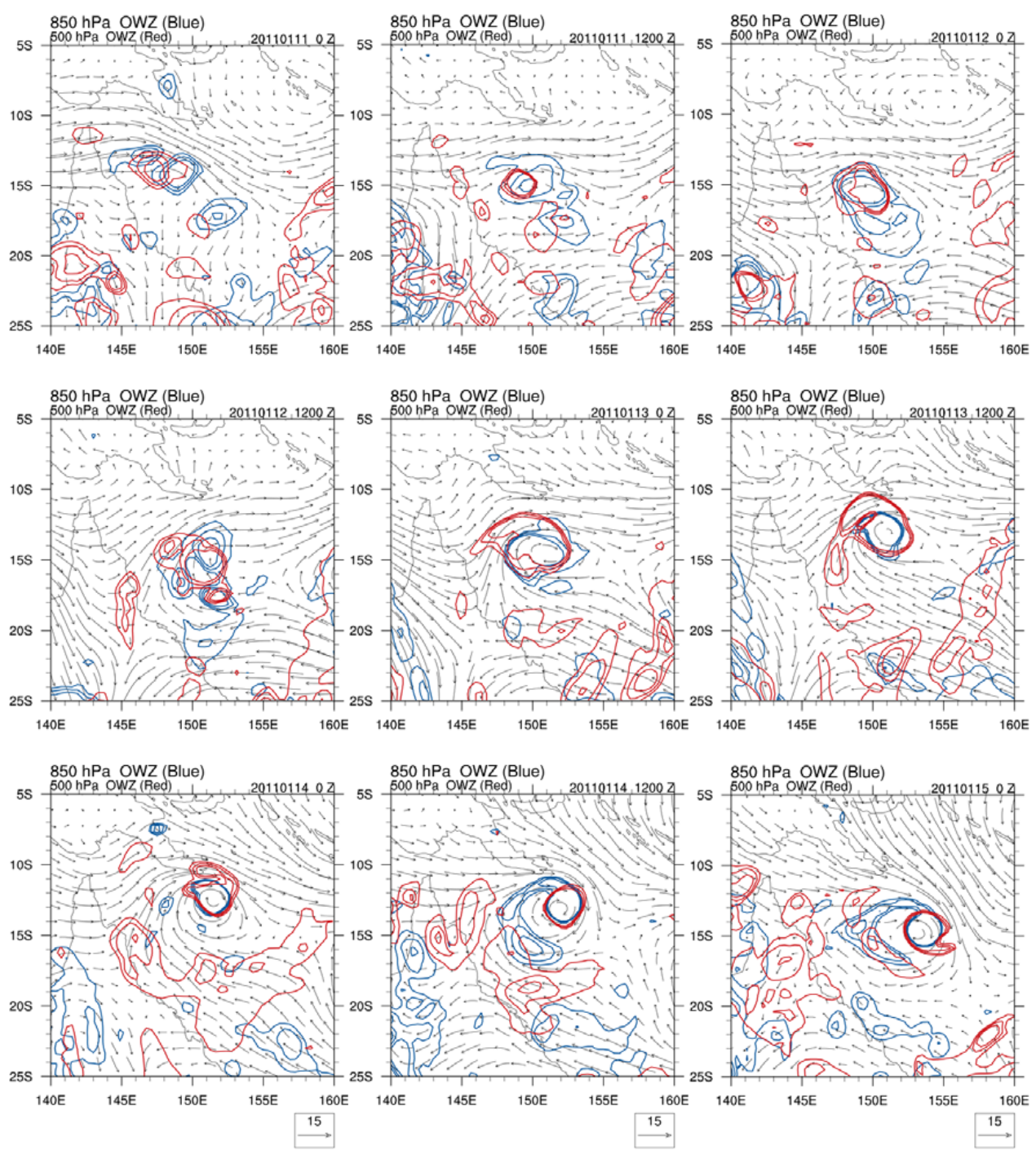

Fig. 5. ACCESS analysis OWZ on the $850 \mathrm{hPa}$ (blue contours, $60,80,100 \times 10^{-6} \mathrm{~s}^{-1}$ ) and $500 \mathrm{hPa}\left(\right.$ red contours, $50,70,90 \times 10^{-6} \mathrm{~s}^{-1}$ ) pressure levels, and $850 \mathrm{hPa}$ horizontal winds (vectors), at 12 hourly intervals for the $72 \mathrm{~h}$ leading up to the formation of TC Zelia (January 2011) in the Coral Sea.

coarse grid data, all of the OWZP ingredients can be resolved by coarse grid models, which in theory suggests a suitably tuned OWZP could be applied as a TC detector to climate models without further tuning.

A detection scheme developed and tuned independent of climate models differs from traditional schemes in which the TC definition is effectively adjusted so that the climatology of climate model TC-like circulations best reproduces the observed TC climatology. Whereas the independently developed and tuned TC detector can be verified against individual observed TCs as well as the observed TC climatology. There are pros and cons for both methods. The traditional method should always provide the most realistic climatology, but it will never be clear how much error compen- sation has been built into the detector between the climate model TC error and the TC detection definition error ${ }^{10}$. This error compensation should be minimal in an independently

\footnotetext{
${ }^{10}$ Climate model TC definitions, derived to overcome issues of under-resolved climate model TCs, may vary between TC detection routines, models and model parameterizations (e.g., Vitart et al., 1997; Yokoi and Tabayaka, 2009; Yokoi et al., 2009), model resolution (e.g., Bengtsson et al., 1995; Murakami and Sugi, 2010), and often between TC basins within individual models (e.g., Camargo and Zebiak, 2002). Because there is no way to directly verify climate model TCs there is no way to directly verify detection algorithms applied to climate models. Thus tuning of detection algorithms to match TC climatology leads to some unknown proportion of model error being absorbed in the subjective choice of TC definition built into the detection algorithm.
} 
developed TC detector tuned to actual observed TCs, but projection biases could be introduced when applied to climate models if systematic differences exist between the modelling systems used to develop and apply the detector.

With the above in mind the OWZP thresholds were adjusted to maximise performance using direct verification in $20 \mathrm{yr}$ of ERA-interim reanalysis data, as described in Sect. 3. We began with the thresholds described in Sect. 4.2, which were somewhat arbitrarily chosen, and with a bit of tuning the performance of the OWZP method as a TC detector was significantly improved. The threshold settings described in Sect. 4.2 yielded about an $85 \%$ hit rate, $(15 \%$ miss rate) but the false alarm rate was quite high at $55 \%$. Up to that point only a token effort had been made to minimise false alarms at higher latitudes. Raising the $950 \mathrm{hPa}$ specific humidity core threshold from 10 to $12.3 \mathrm{~g} \mathrm{~kg}^{-1}$ (OWZP4) eliminated most of the higher latitude false alarms at the small cost of 4 additional misses $(0.26 \%)$, which dropped the false alarm rate to below $50 \%$.

Our experimentation (not shown) demonstrated that the performance increased with increasing OWZ thresholds, but appeared to be optimal with the existing core RH and windshear thresholds. Increasing the $850 \mathrm{hPa}$ core OWZ threshold from 50 to $60 \times 10^{-6} \mathrm{~s}^{-1}$ and the $500 \mathrm{hPa}$ core OWZ threshold from 40 to $50 \times 10^{-6} \mathrm{~s}^{-1}$ (OWZP5) yielded a false alarm rate of $25.7 \%$, and a miss rate of $22.0 \%$ (hit rate of $78 \%$ ). A preliminary indication of the OWZP5 performance as a TC detector in ERA-interim reanalysis data is illustrated in Fig. 6, which shows the observed and detected TC formation climatology for 1989-2008. The method reproduces the regional and interannual TC climatology very well. The limited comparisons we are able to make with other TC detection schemes applied to reanalysis data, suggest the OWZP detection method performs equally well or better than two other schemes. A much more detailed performance assessment will appear in a future paper.

\section{Summary}

The importance of recirculating flow in quasi-closed circulations for the development of tropical cyclones in tropical waves has been the subject of a number of recent studies (Dunkerton et al., 2009; Montgomery et al., 2010; Raymond and López Carrillo, 2011). The quasi-circulation contains an enhanced vortical environment that provides the background vorticity from which the TC vortex is constructed, and it offers protection from the intrusion of dry air that might disrupt the developing circulation. A thermodynamic transformation that occurs near the centre of the quasi-closed circulation (Wang, 2012), where there is strong rotation and weak deformation, favours deep and broad vigorous convection capable of driving a large inward mass flux in the low- to midtroposphere.
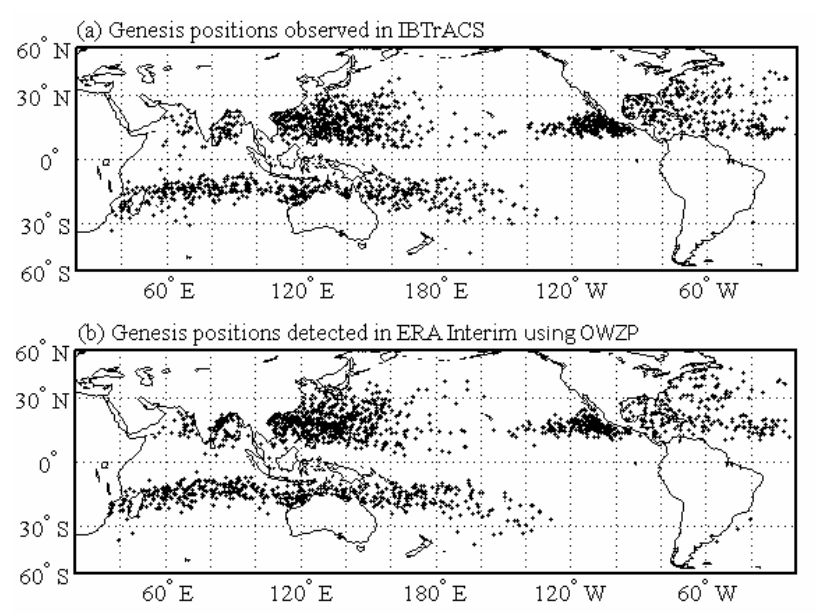

Fig. 6. TC formation locations (a) observed in IBTrACS, and (b) detected in the ERA-interim reanalysis data using OWZP5 (Table 1) for the period 1989-2008.

In Sect. 2 it was argued that in an axisymmetric circulation with minimal inflow, such that the system relative streamlines are approximately circular, the outermost streamline of maximum curvature vorticity encloses a region that can most efficiently support vortex intensification, and that the efficiency is greatest for solid body rotation inside that streamline. This region coincides with the strong rotation and weak deformation surrounding the DMW formation sweetspot, and the central pouch region of Wang (2012) where the thermodynamic transformation occurs. A Galilean invariant quantity that effectively measures low-deformation vorticity is introduced as a means of simply identifying the formation sweet-spot in instantaneous model data. The quantity (OWZ, Eq. 10) is essentially the product of absolute vorticity and a normalized Okubo-Weiss parameter (Eq. 8). For solid body rotation the OWZ has the same magnitude as absolute vorticity, and has a value of zero for pure shear vorticity or for flows in which deformation exceeds the vorticity.

While the DMW papers have demonstrated the importance of wave-relative quasi-closed circulations for TC formation in tropical waves, there has been less attention on other TC formation environments. In this paper we ask the question whether all TCs form in regions of enhanced vorticity with weak deformation. By monitoring the OWZ signatures of pre-formation circulations in ERA-interim reanalysis data we arrived at a set of favourable dynamic conditions for TC formation. These include enhanced OWZ on the 850 and $500 \mathrm{hPa}$ levels with some vertical overlap, in an environment of relatively weak vertical wind shear. In the $20 \mathrm{yr}$ of ERA-interim data analysed (1989-2008) about $90 \%$ of TCs identified in the IBTrACS global database showed the above dynamic conditions were satisfied for at least $24 \mathrm{~h}$ prior to the TC declaration. Given the subjectivity surrounding TC declarations, the issues of data consistency and quality throughout 
the global TC basins, and the relatively coarse resolution of the reanalysis data, this number is encouragingly high, and suggests that enhanced OWZ may indeed be necessary for $\mathrm{TC}$ formation, and that it is a fairly robust and useful indicator of favourable dynamic genesis environments.

However, this favourable environment must be sustained long enough for the TC gestation to occur. Applying additional thresholds of relative humidity to ascertain the state of the thermodynamic transition, and wind shear to determine whether the vortex is developing in a destructive environment, it was found that generally TC formation required all thresholds to be satisfied continuously for about $48 \mathrm{~h}$. This result gives confidence that the combined thresholds (labelled the OWZ parameter, OWZP) can be used as a TC formation diagnostic, and as a TC detector in coarse resolution model data including global climate models. Testing the OWZP as a formation diagnostic is currently underway, and the TC detection application has been developed, tested and applied to a selection of CMIP3 climate models, the results of which will be presented in two future papers.

\section{Appendix A}

\section{Tracker}

As noted in Sect. 3.4 the tracking routine is a five-step process:

i. neighbouring groups of grid points that satisfy the initial thresholds are grouped together to create a single "clump" of grid points,

ii. each clump is assessed to see if it satisfies a set of clump conditions,

iii. clumps in close proximity are reduced to one clump by discarding the weaker or smaller clumps,

iv. storm tracks are constructed by stringing together clumps from consecutive times,

v. storm tracks are assessed to see whether they satisfy an additional track condition.

In step (i) grid points with the initial thresholds satisfied are considered to be neighbours if they are immediate (north, south, east or west) or diagonal neighbours (north-east, south-east, south-west, north-west). Four clump examples are illustrated in Fig. A1. Once a clump is identified it is numbered and assigned a single value for each of the threshold quantities equal to the average grid point value in the clump.

In step (ii) each clump is assessed to see whether it satisfies the clump conditions. The clump conditions require: (1) two or more grid points in the clump to satisfy the core thresholds (Table 1) and be located over the sea; and (2) the clumpaveraged values of $850 \mathrm{hPa}$ OWZ, $500 \mathrm{hPa}$ OWZ, $950 \mathrm{hPa}$

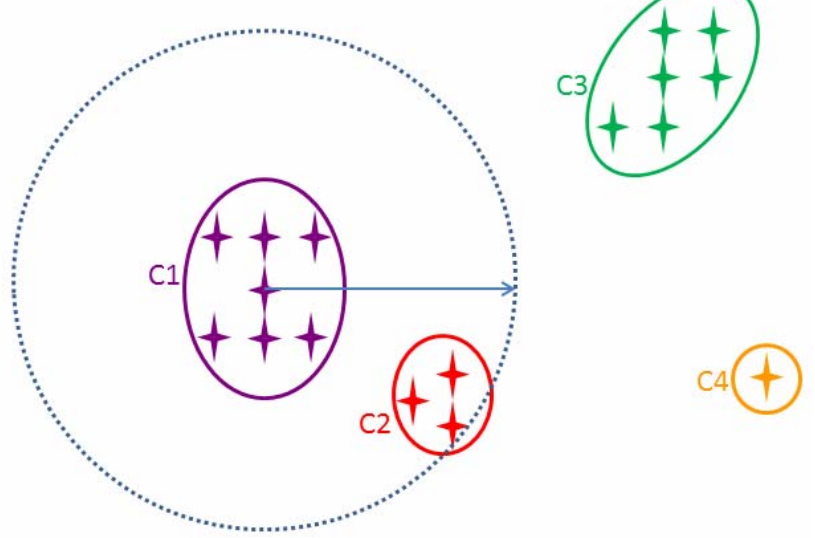

Fig. A1. Schematic illustration of the grid-point clump analysis. Grid points that satisfy the initial thresholds are indicated by stars. Neighbouring grid points are grouped into clumps (circled and labelled C1-C4). Clump 2 lies within a critical radius (dotted line) of Clump 1. It is assumed that they belong to the same circulation, and for simplicity the weaker or smaller clump is discarded (see Fig. A2). Clump 4 contains only one grid point. It will be included in the track construction procedure (Fig. A3) but it will not meet the size requirement of the clump conditions used in this paper.

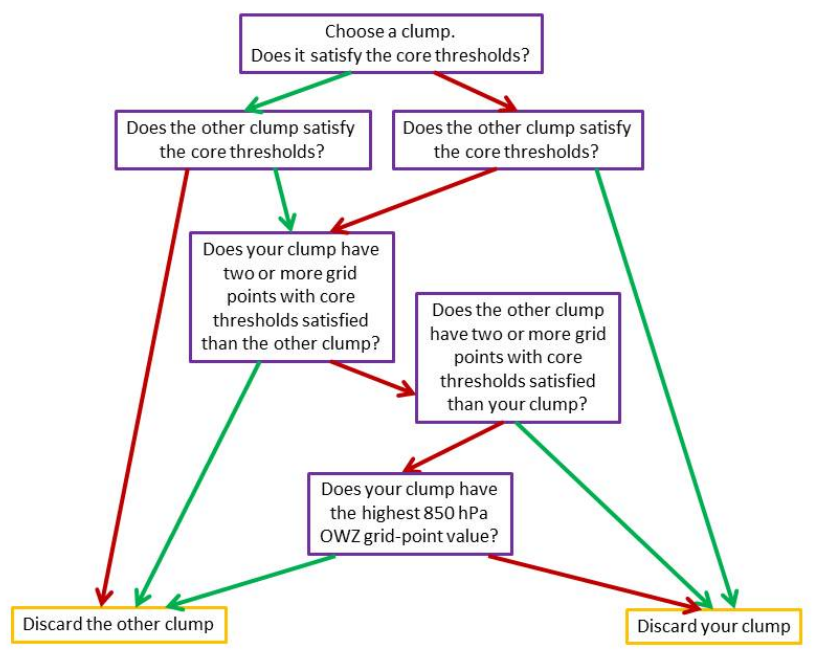

Fig. A2. Flow chart describing the decision making pathway for choosing which of two nearby grid point clumps is to be retained and which is to be discarded. Green arrows represent a yes response to the questions posed and red arrows represent a no response.

$\mathrm{RH}$ and $950 \mathrm{hPa}$ SH must exceed the core thresholds. Clumps that satisfy the clump conditions are flagged as true (T) for the storm-track assessment in step (v).

Often in the early formation phases two or more clumps may be found in relatively close proximity. In almost all cases they can be considered to be a part of the same system, thus it is only necessary to record one clump. In step (iii) a search for nearby clumps is performed as follows. Using 


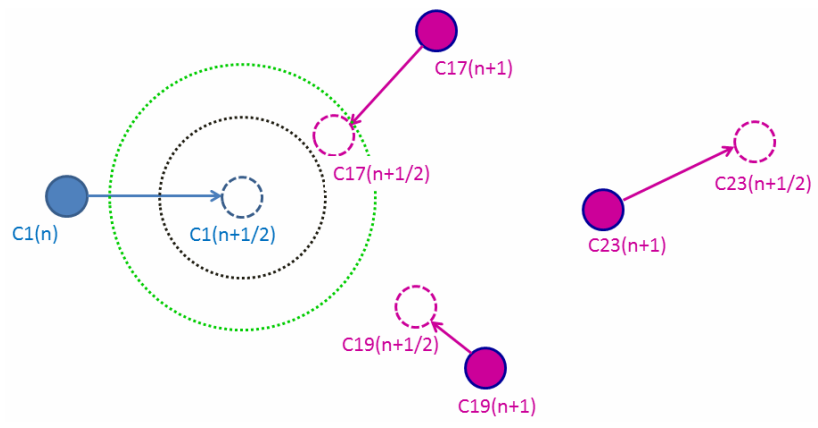

Fig. A3. Schematic illustration of the storm-track construction procedure. Beginning with Clump 1 at time $n$ (blue circle) the average steering velocity from all Clump 1 grid points is used to estimate the Clump 1 position at time $n+1 / 2$ (blue dashed circle). For all clumps at time $n+1$ (purple circles), the average steering velocities are reversed to estimate their positions at time $n+1 / 2$ (purple dashed circles). A search is extended outwards from the estimated half-way position of Clump 1, in $100 \mathrm{~km}$ radius increments, for a potential match with an $n+1$ clump. The first search (black dotted circle) finds no match. The second search (green dotted circle) finds a match with Clump 17. Clump 1 and 17 are considered to be the same circulation separated by one unit of time.

the average latitude and longitude of each grid point in a clump to define the clump position, a search for a nearby clump within a $550 \mathrm{~km}$ radius is performed (e.g., Clump 2 is deemed to be nearby to Clump 1 in Fig. A1). A test is applied to determine which clump is retained and which is discarded. A flow-chart depicting the decision process is given in Fig. A2. If one clump satisfies the clump conditions and the other clump does not, then the former is retained. If both clumps satisfy the clump conditions or both do not satisfy the clump conditions, then the larger or more intense clump is retained. The clump size is deemed to be the number of grid points in the clump that satisfy the core thresholds (Table 1). If one clump is two or more grid points larger than the other clump then it is retained. Otherwise the clump with the greatest grid point $850 \mathrm{hPa} O W Z$ value is retained.

The construction of storm tracks, step (iv), is illustrated in Fig. A3. It involves a complex search routine for future matching clumps. A present-time clump is chosen and its half-way or half-time position is estimated from its steering velocity (i.e., $+12 \mathrm{~h}$ position using $24 \mathrm{~h}$ data). This position is compared with the estimated half-way positions of all clumps from the next time period (i.e., $-12 \mathrm{~h}$ position using $24 \mathrm{~h}$ data). The search first looks for a match within $100 \mathrm{~km}$ and deems the very first match it finds to be the correct "link" in the storm track. If no match is found the search is repeated at increasingly larger radii (100 km increments) until a latitude-dependent maximum search radius (defined below) is reached. The search progresses forwards in time as each link is found until a link can no longer be found at the next time period. However, before abandoning this particular track an additional search is made two time periods

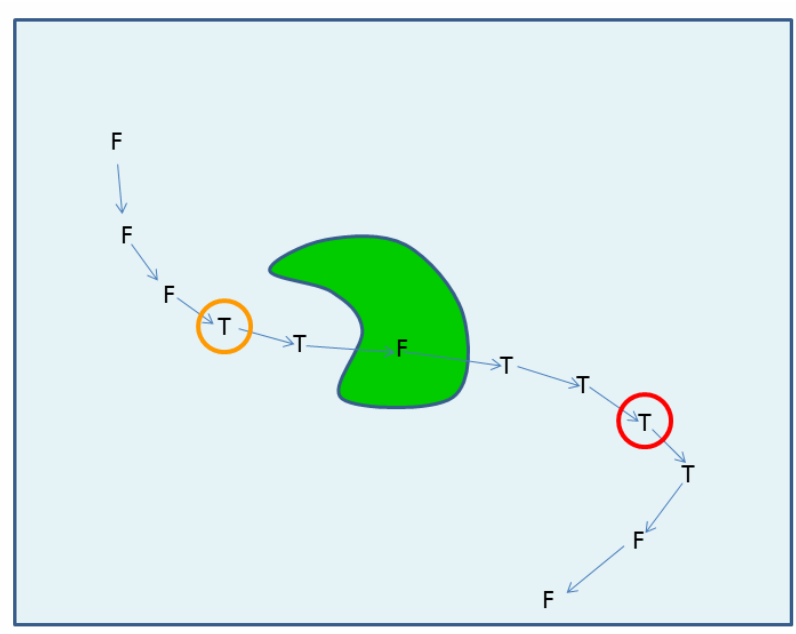

Fig. A4. Schematic illustration of the storm-track condition assessment procedure. Each clump is individually assessed and is flagged true $(\mathrm{T})$ or false $(\mathrm{F})$ depending on whether it satisfies the clump conditions. The storm-track condition specifies a minimum number of consecutive true clumps. The orange and red circles indicate the storm position after 1 and 3 consecutive true clumps respectively.

in advance, by estimating half way positions $\pm 24 \mathrm{~h}$. This was found to be important for tracking both mature storms (when occasionally the wind shear threshold was temporarily exceeded) and young systems, when the thresholds may be borderline. The track is terminated when no match is found two time periods in advance. Once all tracks have been constructed any isolated clumps are assumed to be associated with very short-lived circulations and are discarded.

The latitude-dependent search radius varies linearly from 600 to $400 \mathrm{~km}$ between $15^{\circ}$ and $30^{\circ}$ latitude in both hemispheres, with constant values outside this latitude band. The latitude dependent search radius was found to be necessary due to numerous circulations in closer proximity at the higher latitudes.

In step (v) the tracks are tested to see whether the track condition, of consecutive clumps satisfying the core clump conditions, is met. Figure A4 shows a hypothetical track that passes over an island. The clumps that satisfied the clump conditions of step (iii) are labelled $\mathrm{T}$ (true) and the others labelled $\mathrm{F}$ (false). For example, the orange and red circles could indicate the location of the storm when the track conditions are met for experiments OWZP2 and OWZP3 (Table 1) respectively. 
Acknowledgements. We thank Tim Dunkerton, Kevin Walsh, Joe Courtney, Peter Otto, Zhuo Wang, Jeff Kepert and an anonymous reviewer for their very useful suggestions for improving the manuscript. We acknowledge the Pacific Climate Change Science Project (PCCSP) for supporting this work.

Edited by: T. J. Dunkerton

\section{References}

Bengtsson, L., Botzet, M., and Esch, M.: Hurricane-type vortices in a general circulation model, Tellus, 47A, 175-196, 1995.

Camargo, S. J. and Zebiak, S. E.: Improving the detection and tracking of tropical storms in atmospheric general circulation models, Weather Forecast., 17, 1152-1162, 2002.

Camargo, S. J., Sobel, A. H., Barnston, A. G., and Emanuel, K. A.: Tropical cyclone genesis potential index in climate models, Tellus, 59A, 428-443, 2007.

Charney, J. G. and Eliasen, A.: On the growth of the hurricane depression, J. Atmos. Sci., 21, 68-75, 1964.

Cruz Gómez, R. C. and Bulgakov, S. N.: Remote sensing observations of the coherent and non-coherent ring structures in the vicinity of Lesser Antilles, Ann. Geophys., 25, 331-340, doi:10.5194/angeo-25-331-2007, 2007.

Davidson, N. E., Holland, G., McBride, J. L., and Keenan, T. D.: On the formation of AMEX Tropical Cyclones Irma and Jason, Mon. Weather Rev., 118, 1981-2000, 1990.

Dee, D. P., Uppala, S. M., Simmons, A. J., Berrisford, P., Poli, P., Kobayashi, S., Andrae, U., Balmaseda, M. A., Balsamo, G., Bauer, P., Bechtold, P., Beljaars, A. C. M., van de Berg, L., Bidlot, J., Bormann, N., Delsol, C., Dragani, R., Fuentes, M., Geer, A. J., Haimberger, L., Healy, S. B., Hersbach, H., Hólm, E. V., Isaksen, L., Kållberg, P., Köhler, M., Matricardi, M., McNally, A. P., Monge-Sanz, B. M., Morcrette J.-J., Park, B.-K., Peubey, C., de Rosnay, P., Tavolato, C., Thépaut, J.-N., and Vitart, F.: The ERA-Interim reanalysis: configuration and performance of the data assimilation system, Quart. J. Roy. Meteor. Soc., 137, 553-597, doi:10.1002/qj.828, 2011.

Davis, C. A. and Ahijevych, D. A.: Mesoscale structural evolution of three tropical weather systems observed during PREDICT, J. Atmos. Sci., 69, 1284-1305, 2012.

Dunkerton, T. J., Montgomery, M. T., and Wang, Z.: Tropical cyclogenesis in a tropical wave critical layer: easterly waves, Atmos. Chem. Phys., 9, 5587-5646, doi:10.5194/acp-9-5587-2009, 2009.

Emanuel, K. A.: Atmospheric convection, Oxford University Press, 1994.

Emanuel, K. A. and Nolan, D. S.: Tropical cyclone activity and the global climate system. Preprints, 26th Conf. on Hurricanes and Tropical Meteorology, Miami, FL, Amer. Meteor. Soc., 240-241, 2004.

Gray, W. M.: Global view of the origin of tropical disturbances and storms, Mon. Weather Rev., 96, 669-700, 1968.

Gray, W. M.: Cumulus convection and larger scale circulations. I. Broadscale and mesoscale considerations, Mon. Weather Rev., 101, 839-855, 1973.

Gray, W. M.: Hurricanes: their formation structure, and likely role in the tropical circulation. Meteorology over tropical oceans, edited by: Shaw, D. B., Roy. Meteor Soc., 155-218, 1979.
Hack, J. J. and Schubert, W. H.: Nonlinear response of atmospheric vortices to heating by organized cumulus convection, J. Atmos. Sci., 43, 1559-1573, 1986.

Haller, G. and Yuan, G.: Lagrangian coherent structures and mixing in two-dimensional turbulence, Physica D, 147, 352-370, 2000.

Harper, B. A., Kepert, J. D., and Ginger, J. D.: Guidelines for converting between various wind averaging periods in tropical cyclone conditions, World Meteorological Organization, WMO/TD-No. 1555, 2010.

Hendricks, E. A., Montgomery, M. T., and Davis, C. A.: On the role of "vortical" hot towers in hurricane formation, J. Atmos. Sci., 61, 1209-1232, 2004.

Holton, J. R.: An introduction to dynamic meteorology, Elsevier Academic Press, Burlington, MA, 535 pp., 2004.

Isern-Fontanet, J., Garcia-Ladona, E., and Font, J.: Vortices of the Mediterranean Sea: An Altimetric Perspective, J. Phys. Oceaonog., 36, 87-103, 2006.

Kingsmill, D. E. and Houze Jr., R. A.: Thermodynamic characteristics of air flowing into and out of precipitating convection over the west Pacific warm pool, Quart. J. Roy. Meteor. Soc., 125, 1209-1229, 1999.

Knapp, K. R. and Kruk, M. C.: Quantifying Interagency Differences in Tropical Cyclone Best-Track Wind Speed Estimates, Mon. Weather Rev., 138, 1459-1473, 2010.

Knapp, K. R., Kruk, M. C., Levinson, D. H., and Gibney, E. J.: Archive compiles new resource for global tropical cyclone research, Eos, Transactions, AGU, 90, 45-49, doi:10.1029/2009EO060002, 2009.

Mapes, B. E. and Houze, R. A.: An integrated view of the 1987 Australian monsoon and its mesoscale convective systems. I: Horizontal structure, Quart. J. Roy. Meteor. Soc., 118, 927-963, 1992.

Mapes, B. E. and Houze, R. A.: An integrated view of the Australian monsson and its mesoscale convective systems. Part II: Vertical structure, Quart. J. Roy. Meteor. Soc., 119, 733-754, 1993.

Mapes, B. E. and Houze, R. A.: Diabatic divergent profiles in Western Pacific Mesoscale Convective Systems, J. Atmos. Sci., 52, 1807-1828, 1995.

Martin, G. M., Ringer, M. A., Pope, V. D., Jones, A., Dearden, C., and Hinton, T. J.: The physical properties of the atmosphere in the new Hadley Centre Global Environmental Model (HadGEM1). Part I: Model description and global climatology, J. Clim., 19, 1274-1301, doi:10.1175/JCLI3636.1, 2006.

McBride, J. L. and Zehr, R.: Observational analysis of tropical cyclone formation. Part II: Comparison of non-developing versus developing systems, J. Atmos. Sci., 38, 1132-1151, 1981.

Menkes, C. E., Lengaigne, M., Marchesiello, P., Jourdain, N. C., Vincent, E. M., Lefèvre, J., Chauvin, F., and Royer, J.-F.: Comparison of tropical cyclogenesis indices on seasonal to interannual timescales. Climate Dyn., 38, 301-321, doi:10.1007/s00382-011-1126-x, 2011.

Montgomery, M. T., Nicholls, M. E., Cram, T. A., and Saunders, A.: A vortical hot tower route to tropical cyclogenesis, J. Atmos. Sci., 63, 355-386, 2006.

Montgomery, M. T., Lussier III, L. L., Moore, R. W., and Wang, Z.: The genesis of Typhoon Nuri as observed during the Tropical Cyclone Structure 2008 (TCS-08) field experiment - Part 1: The role of the easterly wave critical layer, Atmos. Chem. Phys., 10, 9879-9900, doi:10.5194/acp-10-9879-2010, 2010. 
Montgomery, M. T., Davis, C., Dunkerton, T., Wang, Z., Velden, C., Torn, R., Majumdar, S. J., Zhang, F., Smith, R. K., Bosart, L., Bell M. M., Haase, J. S., Heymsfield, A., Jensen, J., Campos, T., and Boothe, M. A.: The pre-depression investigation of cloudsystems in the tropics (PREDICT) experiment. Scientific basis, new analysis tools and some first results, B. Am. Meteorol. Soc., 93, 153-172, 2012.

Murakami, H. and Sugi, M.: Effect of model resolution on tropical cyclone climate projections, SOLA, 6, 73-76, 2010.

Nolan, D. S.: What is the trigger for tropical cyclogenesis?, Aus. Met. Mag., 56, 241-266, 2007.

Okubo, A.: Horizontal dispersion of floatable particles in the vicinity of velocity singularities such as convergences, Deep-Sea Res., 17, 445-454, 1970.

Provenzale, A.: Transport by coherent barotropic vortices, Annu. Rev. Fluid Mech., 31, 55-93, 1999.

Puri, K., Dietachmayer, G., Steinle, P., Dix, M., Rikus, L., Logan, L., Naughton, M., Tingwell, C., Xiao, Y., Barras, V., Bermous, I., Bowen, R., Deschamps, L., Franklin, C., Fraser, J., Glowacki, T., Harris, B., Lee, J., Le, T., Roff, G., Sulaiman, A., Sims, H., Sun, X., Sun, Z., Zhu, H., Chattopadhyay, M., and Engel, C.: Implementation of the initial ACCESS Numerical Weather Prediction system, Aus. Met. Ocean. J., in press, 2013.

Raymond, D. J. and López Carrillo, C.: The vorticity budget of developing typhoon Nuri (2008), Atmos. Chem. Phys., 11, 147163, doi:10.5194/acp-11-147-2011, 2011.

Riehl, H. and Malkus, J. S.: On the heat balance in the equatorial trough zone, Geophysica, 6, 503-538, 1958.

Royer, J. F., Chauvin, F., Timbal, B., Araspin, P., and Grimal, G.: A GCM study of the impact of greenhouse gas increase on the frequency of occurrence of tropical cyclones, Climatic Change, 38, 307-343, 1998.

Rozoff, C., Schubert, W., Brian, D., and Kossin, J.: Rapid Filamentation Zones in Intense Tropical Cyclones, J. Atmos. Sci., 63, 325-340, 2006.

Schubert, W. H. and Hack, J. J.: Inertial stability and tropical cyclone development, J. Atmos. Sci., 39, 1687-1697, 1982.

Shapiro, L. J. and Willoughby, H.: The response of balanced hurricanes to local sources of heat and momentum, J. Atmos. Sci., 39, 378-394, 1982.

Tippett, M. K., Camargo, S. J., and Sobel, A. H.: A Poisson Regression Index for Tropical Cyclone Genesis and the Role of LargeScale Vorticity in Genesis, J. Climate, 24, 2335-2357, 2011.

Tory, K. J. and Frank, W. M.: Tropical cyclone formation. Chapter 2: Global Perspectives on Tropical Cyclones, Second Edition, edited by: Chan, J. and Kepert, J. D., World Scientific, 2010.
Tory, K. J., Montgomery, M. T., Davidson, N. E., and Kepert, J. D.: Prediction and diagnosis of Tropical Cyclone formation in an NWP system. Part II: A diagnosis of tropical cyclone Chris formation, J. Atmos. Sci., 63, 3091-3113, 2006.

Tory, K. J., Montgomery, M. T., and Davidson, N. E.: Prediction and diagnosis of Tropical Cyclone formation in an NWP system. Part III: Developing and non-developing storms, J. Atmos. Sci., 64, 3195-3213, 2007.

Vitart, F., Anderson, J. L., and Stern, W. F.: Simulation of interannual variability of tropical storm frequency in an ensemble of GCM integrations, J. Climate, 10, 745-760, 1997.

Wang, Y.: Rapid Filamentation Zone in a Numerically Simulated Tropical Cyclone, J. Atmos. Sci., 65, 1158-1181, 2008.

Wang, Z.: Thermodynamic aspects of tropical cyclone spin-up, J. Atmos. Sci., 69, 2433-2451, 2012.

Wang, Z., Montgomery, M. T., and Dunkerton, T. J.: A dynamicallybased method for forecasting tropical cyclogenesis location in the Atlantic sector using global model products, Geophys. Res. Lett., 36, L03801, doi:10.1029/2008GL035586, 2009.

Wang, Z., Montgomery, M. T., and Dunkerton, T. J.: Genesis of Prehurricane Felix (2007). Part I: The role of the wave critical layer, J. Atmos. Sci., 67, 1711-1729, 2010a.

Wang, Z., Montgomery, M. T., and Dunkerton, T. J.: Genesis of Prehurricane Felix (2007). Part II: Warm core formation, precipitation evolution and predictability, J. Atmos. Sci., 67, 1730-1744, 2010 b.

Wang, Z., Montgomery, M. T., and Fritz, C.: A First Look at the Structure of the Wave Pouch during the 2009 PREDICT-GRIP Dry Runs over the Atlantic, Mon. Weather Rev., 140, 1144-1163, 2012.

Weiss, J.: The dynamics of enstrophy transfer in two-dimensional hydrodynamics, Phys. D, 48, 273-294, 1991.

Yanai, M., Esbensen, S., and Chu, J.-H.: Determination of bulk properties of tropical cloud clusters from large-scale heat and moisture budgets, J. Atmos. Sci., 30, 611-627, 1973.

Yokoi, S. and Takayabu, Y. N.: Multi-model projection of global warming impact on tropical cyclone genesis frequency over the western North Pacific, J. Meteor. Soc. Japan, 87, 525-538, 2009.

Yokoi, S., Takayabu, Y. N., and Chan, J. C. L.: Tropical cyclone genesis frequency over the western North Pacific simulated in medium resolution coupled general circulation models, Clim. Dyn., 33, 665-538, 2009.

Zehr, R.: Tropical cyclogenesis in the western North Pacific, NOAA Tech. Rep. NESDIS 61, 181 pp., 1992. 